\title{
Dual RNASeq Reveals NTHi- Macrophage Transcriptomic Changes During Intracellular Persistence
}

\author{
Jodie Ackland ${ }^{1}$, Ashley I. Heinson ${ }^{1}$, David W. Cleary ${ }^{1,2}$, Myron Christodoulides ${ }^{1}$, \\ Tom M. A. Wilkinson ${ }^{1,2,3}$ and Karl J. Staples ${ }^{1,2,3^{*}}$ \\ ${ }^{1}$ Clinical and Experimental Sciences, Faculty of Medicine, University of Southampton, Southampton, United Kingdom, \\ ${ }^{2}$ NIHR Southampton Biomedical Research Centre, University Hospital Southampton NHS Foundation Trust, \\ Southampton, United Kingdom, ${ }^{3}$ Wessex Investigational Sciences Hub, Southampton General Hospital, Faculty of \\ Medicine, University of Southampton, Southampton, United Kingdom
}

OPEN ACCESS

Edited by:

Matthew C. Wolfgang,

University of North Carolina at

Chapel Hill, United States

Reviewed by:

Brian Patrick Conlon,

University of North Carolina at

Chapel Hill, United States

Raja Veerapandian,

Texas Tech University Health Sciences

Center El Paso, United States

*Correspondence:

Karl J. Staples

K.Staples@soton.ac.uk

Specialty section:

This article was submitted to

Bacteria and Host,

a section of the journal

Frontiers in Cellular and

Infection Microbiology

Received: 10 June 2021

Accepted: 26 July 2021

Published: 23 August 2021

Citation:

Ackland J, Heinson Al, Cleary DW, Christodoulides M, Wikinson TMA and Staples KJ (2021) Dual RNASeq

Reveals NTHi-Macrophage

Transcriptomic Changes During

Intracellular Persistence.

Front. Cell. Infect. Microbiol. 11:723481.

doi: 10.3389/fcimb.2021.723481
Nontypeable Haemophilus influenzae (NTHi) is a pathobiont which chronically colonises the airway of individuals with chronic respiratory disease and is associated with poor clinical outcomes. It is unclear how NTHi persists in the airway, however accumulating evidence suggests that NTHi can invade and persist within macrophages. To better understand the mechanisms of NTHi persistence within macrophages, we developed an in vitro model of NTHi intracellular persistence using human monocyte-derived macrophages (MDM). Dual RNA Sequencing was used to assess MDM and NTHi transcriptomic regulation occurring simultaneously during NTHi persistence. Analysis of the macrophage response to NTHi identified temporally regulated transcriptomic profiles, with a specific 'core' profile displaying conserved expression of genes across time points. Gene list enrichment analysis identified enrichment of immune responses in the core gene set, with KEGG pathway analysis revealing specific enrichment of intracellular immune response pathways. NTHi persistence was facilitated by modulation of bacterial metabolic, stress response and ribosome pathways. Levels of NTHi genes bioC, mepM and $d p s$ were differentially expressed by intracellular NTHi compared to planktonic NTHi, indicating that the transcriptomic adaption was distinct between the two different NTHi lifestyles. Overall, this study provides crucial insights into the transcriptomic adaptations facilitating NTHi persistence within macrophages. Targeting these reported pathways with novel therapeutics to reduce NTHi burden in the airway could be an effective treatment strategy given the current antimicrobial resistance crisis and lack of NTHi vaccines.

Keywords: macrophage, NTHi, intracellular persistence, dual RNAseq, host-pathogen interactions

\section{INTRODUCTION}

Haemophilus influenzae is a human-restricted pathobiont (Erwin and Smith, 2007) and is commonly isolated from the nasopharynx, middle ear and respiratory tract (King, 2012; Swords, 2012; Ahearn et al., 2017). H. influenzae can be divided into typeable and nontypeable strains depending on the presence or absence of a polysaccharide capsule. Encapsulated strains are 
classified into six serotypes (a-f), with strains not in possession of a capsule unable to be serotyped and are designated as nontypeable Haemophilus influenzae (NTHi). NTHi is associated with various diseases including pneumonia, meningitis, sinusitis, otitis media and exacerbations of chronic respiratory diseases such as Chronic Obstructive Pulmonary Disease (COPD) and asthma (King, 2012; Finney et al., 2014; Green et al., 2014; Van Eldere et al., 2014; McCann et al., 2016). Although NTHi is implicated in exacerbations of chronic respiratory diseases, NTHi has also been isolated from the airway during stable periods of disease (Wood et al., 2010; Iikura et al., 2015; Zhang et al., 2016; Wilkinson et al., 2017; Mayhew et al., 2018). The duration of NTHi airway colonisation varies, with longitudinal studies suggesting persistence ranges from months up to as long as 7 years (Murphy et al., 2004; Román et al., 2004; Gallo et al., 2018).

NTHi has traditionally been considered an extracellular pathogen, however an increasing number of reports suggest NTHi is able to invade immune cells to enhance airway persistence and survival (Ahrén et al., 2001; Craig et al., 2001; Craig et al., 2002; King et al., 2008; Morey et al., 2011). The predominant innate immune cell in the healthy respiratory tract is the macrophage, which orchestrates the airway immune defence response, regulates inflammation, maintains homeostasis and participates in immune resolution processes (Mosser and Edwards, 2008; Byrne et al., 2015). Despite these key processes, accumulating evidence suggests that macrophages are a target of infection for NTHi. One of the first indications of NTHi invasion of immune cells was provided by Forsgren et al., who used transmission electron microscopy to visualise replicating, intracellular NTHi within macrophage-like cells isolated from adenoid tissue (Forsgren et al., 1994). Subsequent in vitro studies have reported varying mechanisms of NTHi invasion of phagocytic cells including receptor-mediated endocytosis (Ahrén et al., 2001), lipid raft mediated endocytosis (Martí-Lliteras et al., 2009) and phagocytosis (Clementi and Murphy, 2011), with strain-dependent differences between clinical isolates reported (Craig et al., 2001). Despite the prominent role of macrophages in the innate immune response against NTHi, the mechanisms of NTHi intracellular persistence within macrophages are not well understood.

Furthermore, macrophage dysfunction has been demonstrated in chronic respiratory diseases. In asthma, macrophages have an altered phenotype (Staples et al., 2012) and reduced phagocytic activity, which worsens with asthma severity (Liang et al., 2014). Monocyte-derived macrophages from the blood of asthma patients also exhibit impaired phagocytosis, suggesting the phagocytic defect is not limited to the lung (Liang et al., 2014). A similar defect in phagocytic capacity has also been reported in patients with COPD, with reduced uptake of NTHi by both alveolar macrophages and monocyte-derived macrophages (Berenson et al., 2006; Taylor et al., 2010), which is associated with exacerbation frequency (Singh et al., 2021) and disease severity (Berenson et al., 2013). Impairment in macrophage regulation and clearance of NTHi could contribute to persistent and chronic NTHi colonisation of the lung.
Genomic studies have identified NTHi genome evolution during airway persistence (Pettigrew et al., 2018), however RNASeq can offer insights into the dynamic transcriptomic changes which occur during host-pathogen interactions. Previous studies have shown modulation of NTHi gene expression contributes to enhanced intracellular survival within epithelial cells by upregulation of bacterial stress response genes and metabolic pathways (Craig et al., 2002; Baddal et al., 2015). It is not clear whether NTHi similarly modulates gene expression during intracellular persistence of macrophages, enabling NTHi survival and evasion of macrophage immune responses to facilitate chronic airway colonisation. Given the challenges associated with developing an efficient NTHi vaccine, identifying how NTHi can persist within the airway could be crucial in guiding the development of antimicrobial therapeutics aimed at reducing NTHi burden in chronic respiratory disease. Although the initial stages of NTHi invasion and entry into macrophages have been documented (Ahrén et al., 2001; Martí-Lliteras et al., 2009; Clementi and Murphy, 2011), it is not clear how NTHi is able to survive once inside a macrophage. Therefore, the aim of this work was to investigate NTHi-macrophage interactions using dual RNASeq to determine transcriptomic changes during intracellular persistence.

\section{MATERIALS AND METHODS}

\section{MDM Culture and Infection}

Blood from healthy volunteers was collected in accordance with the protocol as approved by the Hampshire A Research Ethics Committee (13/SC/0416). Monocytes isolated from blood were seeded at $5 \times 10^{5}$ cells per well and differentiated for $12 \mathrm{~d}$ as previously described (Staples et al., 2012; Cooper et al., 2018). On day 12 , culture media was replaced with reduced serum $(0.1 \%$ foetal bovine serum, FBS), antibiotic-free RPMI media and either left uninfected or were challenged with NTHi at multiplicity of infection (MOI) 100 for $6 \mathrm{~h}$. At $6 \mathrm{~h}$, media was removed and cells washed twice before addition of reduced serum RPMI supplemented with $500 \mu \mathrm{g} / \mathrm{ml}$ gentamicin to wells for $90 \mathrm{~min}$ in order to kill and remove extracellular bacteria (referred to as ' 6 h time point'). Gentamicin-containing RPMI media was removed and cells were washed before further incubation in reduced serum RPMI media until harvest at $24 \mathrm{~h}$ (referred to as ' $24 \mathrm{~h}$ time point'). Presence of NTHi at this $24 \mathrm{~h}$ time point in the absence of antibiotics is regarded as persistence.

\section{NTHi Culture and Growth}

NTHi strains ST14, ST408 and ST201 isolated from COPD patients undergoing bronchoscopy (Osman et al., 2018, Wallington et al., 2018), and NTHi-GFP- $375^{\text {SR }}$ (kindly gifted by Dr. Derek Hood, MRC Harwell, UK) (Mulay et al., 2016) were grown as previously described (Kirkham et al., 2013). Briefly, NTHi was cultured from frozen stock on to chocolate blood agar plates (CHOC, Oxoid, Basingstoke, UK) and incubated overnight at $37^{\circ} \mathrm{C}, 5 \% \mathrm{CO}_{2}$. Single NTHi colony forming units (CFU) were selected and inoculated in supplemented Brain 
Heart Infusion (BHI) media (30 mg/L Hemin (Sigma-Aldrich, Paisley, UK), $10 \mathrm{mg} / \mathrm{L} \beta$-Nicotinamide adenine dinucleotide $\left(\beta-N A D\right.$, Sigma) and $44 \mathrm{ml} / \mathrm{L}$ glycerol at $37^{\circ} \mathrm{C}, 5 \% \mathrm{CO}_{2}$ for $8 \mathrm{~h}$ to achieve mid-log phase. Heat inactivated FBS (20\%) was added to culture and $1 \mathrm{ml}$ aliquots were stored at $-80^{\circ} \mathrm{C}$ until required. Counts of frozen NTHi stocks were routinely performed to determine the concentration of aliquots and to ensure NTHi viability remained stable over time. NTHi aliquots for infection were defrosted and transferred to a fresh tube containing $500 \mu \mathrm{l}$ PBS and centrifuged at $800 \mathrm{~g}$ for $10 \mathrm{~min}, 4^{\circ} \mathrm{C}$ to pellet the bacteria. The remaining supernatant was discarded and the pellet was resuspended in reduced serum, antibiotic-free RPMI media and added to MDM. For assessment of gene expression in planktonic state NTHi, NTHi was prepared for infection as above, but the cell pellet was not resuspended in RPMI and added to MDM and instead was treated with TRIzol reagent (Life Technologies).

\section{Quantifying Intracellular NTHi Persistence}

To enumerate NTHi recovered from MDM, MDM were lysed with $1 \times$ PermWash $^{\text {TM }}$ (PW, BD Biosciences) for 20 min. Cells were serially diluted and plated on $\mathrm{CHOC}$ agar plates (Oxoid) and incubated at $37^{\circ} \mathrm{C}, 5 \% \mathrm{CO}_{2}$ overnight.

\section{Visualisation of NTHi Persistence}

MDM were challenged with GFP- $375^{\mathrm{SR}}$ as described and cells were harvested using Cell Dissociation Solution (Sigma) for 20 min at $37^{\circ} \mathrm{C}$. The cell suspension was recovered and centrifuged to generate a cell pellet. The cell pellet was resuspended in PBS and streaked onto PolyFrost Microslides (Solmedia, Shrewsbury, UK). Once dry, slides were fixed using $4 \%$ paraformaldehyde (PFA) for 15 min. Excess PFA was removed and slides were washed in PBS and left until completely dried. Once dry, $25 \mu \mathrm{l}$ Vectashield $^{\circledR}$ Mounting Medium solution containing DAPI nuclear stain $(1.5 \mu \mathrm{g} / \mathrm{ml}$ ) (Vector Laboratories, Inc. Burlingame, CA) was added to each spot and a glass coverslip mounted on top. Slides were visualised using Axioscope KS400 fluorescence microscope using Carl Zeiss Axioscope 3.0 software.

\section{Dual RNASeq}

RNA extraction for sequenced samples was performed using a miRNeasy kit (QIAGEN ${ }^{\circledR}$, Manchester, UK), including a DNase I (QIAGEN ${ }^{\circledR}$ ) treatment step, according to the manufacturer's instructions. RNA quality was assessed using an Agilent Bioanalyzer 2100 system, prior to sequencing of ribosomal RNA-depleted RNA (Illumina ${ }^{\circledR}$ Ribo-Zero Plus rRNA Depletion Kit), which was performed by Novogene (Hong Kong). Libraries were generated using the NEBNext ${ }^{\circledR}$ Ultra $^{\mathrm{TM}}$ Directional RNA Library Prep Kit for Illumina ${ }^{\circledR}$ (NEB, USA). Library quality was assessed on the Agilent Bioanalyzer 2100 system and quantified using a Qubit 2.0 fluorometer (Life Technologies). Sequencing was performed using NovaSeq 6000 Illumina ${ }^{\circledR}$ platform and 150-base pair (bp) paired end reads were generated using a sequencing depth of 90 million reads. Reads containing adapter sequences, poly- $\mathrm{N}$ and low quality reads were removed to obtain clean reads. Mapping of the cleaned, raw data to the respective reference genomes separated MDM and NTHi transcripts in silico. The Spliced Transcripts Alignment to a Reference (STAR) (Dobin et al., 2013) software (version 2.5) was used to map reads to the human genome (hg38) and Bowtie2 (Langmead and Salzberg, 2012) (version 2.2.3) was used to map reads to the NTHi ST14 genome. Aligned reads were quantified using HTSeq (Anders et al., 2015) and data were filtered to remove lowly expressed reads prior to differential gene expression analysis. Two packages, edgeR (Robinson et al., 2009) and DESeq2 (Anders and Huber, 2010), were used to determine changes in host gene expression between uninfected or NTHi-challenged MDM at $6 \mathrm{~h}$ or $24 \mathrm{~h}$ or to determine changes in NTHi gene expression between $6 \mathrm{~h}$ and $24 \mathrm{~h}$. Significantly differentially expressed genes (DEGs) were determined as $\log _{2} \mathrm{FC} \pm 2(\mathrm{MDM})$ or $\log _{2} \mathrm{FC} \pm 1$ (NTHi) and FDR $p$-value $<0.05$ by both edgeR and DESeq2. To explore the biological relevance of the significant DEGs, gene list enrichment analysis and Kyoto Encyclopaedia of Genes and Genomes (KEGG) pathway analysis was performed using ToppFunn (Chen et al., 2009) and ShinyGo (Ge et al., 2020) using default parameter settings (FDR multiple correction method and enrichment significance cut off level 0.05). Clustering of the top gene ontology terms was performed in Cytoscape using the EnrichmentMap (Merico et al., 2010) and AutoAnnotate (Kucera et al., 2016) plugins.

\section{Strain Comparisons}

To assess NTHi strain diversity, the ParSNP package from the Harvest suite (Treangen et al., 2014) was used to analyse 7 clinical isolates of NTHi. The 86-028NP assembly, (GenBank number CP000057.2), was downloaded from https://www.ncbi. nlm.nih.gov/nuccore to be used as a reference. Default parameters were used to construct an assembly-based coreSNP phylogeny. From this analysis, three strains isolated from different anatomical locations and identified on three different clades of the constructed phylogenetic tree were chosen to infect MDM as described for strain comparison infection experiments.

\section{RNA Isolation and qPCR}

Samples were treated with TRIzol reagent (Life Technologies) and RNA was isolated according to manufacturer's instructions. Reverse transcription to produce cDNA was carried out according to the manufacturer's instructions using a High Capacity cDNA Reverse Transcription Kit (Life Technologies) with random hexamers. Quantitative PCR (qPCR) was performed using TaqMan universal PCR master mix, with all primers obtained from Applied Biosystems (Table S3). The qPCR reactions were performed at $95^{\circ} \mathrm{C}$ for $10 \mathrm{~min}$ and 40 cycles of $95^{\circ} \mathrm{C}$ for $15 \mathrm{~s}$ and $60^{\circ} \mathrm{C}$ for 1 min using a $7900 \mathrm{HT}$ Fast Real-Time PCR System. Gene expression of target genes were normalised either to B2M (MDM) or rho (NTHi) using the deltadelta Ct method.

\section{Lactate Dehydrogenase Assay (LDH)}

$\mathrm{LDH}$ release into culture supernatants was assessed by the CytoTox $96^{\circledR}$ Non-Radioactive Cytotoxicity Assay according to the manufacturer's instructions (Promega, Madison, USA). Briefly, $50 \mu \mathrm{l}$ of harvested supernatant and $50 \mu \mathrm{l}$ CytoTox $96^{\circledR}$ 
Reagent was added to a 96 well plate and incubated for $30 \mathrm{~min}$ in the dark at room temperature. $50 \mu \mathrm{l}$ of stop solution was added and the absorbance read on a microplate reader at $490 \mathrm{~nm}$ (Multiskan Ascent, Agilent Technologies, Wokingham, UK). Optical Density (OD) reading of a media only control was regarded as background and subtracted from sample values.

\section{Quantification of MDM Mediator Release}

IL-1 $\beta$, IL- 6 and IL- 8 release into cell culture supernatants was assessed by DuoSet ELISA kits, which was carried out according to manufacturer's instructions (R\&D Systems). IL-10, IL-15, IL$17 \mathrm{C}, \mathrm{IL}-36 \beta$ and TNF- $\alpha$ release into cell culture supernatants was assessed by a customised Luminex Human Magnetic Assay according to manufacturer's instructions (R\&D Systems). Further information is supplied in the Supplementary Methods.

\section{Statistics}

Statistical analysis was performed using GraphPad Prism (version 8 GraphPad Software, San Diego, USA) and statistical significance was determined as $p<0.05$. For paired data, Wilcoxon matchedpairs signed rank test between two groups or Friedman test with Dunn's post hoc test for multiple comparison testing between more than two groups for one independent variable were used. For unpaired data, Kruskal-Wallis with Dunn's post hoc test for multiple comparison testing was used.

\section{RESULTS}

\section{NTHi Persists Intracellularly Within Macrophages}

To determine NTHi-macrophage transcriptomic changes during intracellular persistence, NTHi persistence was modelled using a monocyte-derived macrophage (MDM) model that has previously been described to resemble alveolar macrophages (Akagawa et al., 2006; Tudhope et al., 2008; Taylor et al., 2010). However, as a challenging and limiting factor of dual RNASeq is that bacterial RNA can make up less than $1 \%$ of the total RNA in an infected cell (Marsh et al., 2017), it was first important to determine the time point at which the highest amount of intracellular NTHi was present. As such, a timecourse was first performed to assess the ability of NTHi to reside intracellularly. MDM were challenged with a clinical isolate of NTHi (ST14) for $2 \mathrm{~h}, 6 \mathrm{~h}$ or $24 \mathrm{~h}$ followed by a 90 min gentamicin wash to kill and remove extracellular NTHi, resulting in only intracellular NTHi present in the model. Higher levels of NTHi CFU were recovered from MDM after $6 \mathrm{~h}$ compared to both $2 \mathrm{~h}$ (not significant) and $24 \mathrm{~h}(\mathrm{p}=0.04$ - Figure S1). As intracellular NTHi presence was highest at $6 \mathrm{~h}$, the infection model was extended following the $90 \mathrm{~min}$ gentamicin wash. MDM were incubated in antibiotic-free media until $24 \mathrm{~h}$ to assess the ability of the intracellular NTHi to persist (Figure 1A).

At both $6 \mathrm{~h}$ and $24 \mathrm{~h}$, NTHi was detected and quantified by live viable counting, with no significant difference in NTHi CFU between time points indicating the ability of NTHi to persist until at least $24 \mathrm{~h}$ (Figure 1B). Although live NTHi was quantified using CFU, it was important to determine whether NTHi RNA could be detected at these recovered amounts, to ensure NTHi RNA was detectable prior to sequencing. The hel gene encodes for a conserved NTHi outermembrane protein (lipoprotein e) and has previously been used to assess the presence of H.influenzae in clinical samples by PCR (Yadav et al., 2003; Coughtrie et al., 2018). Expression of the NTHi hel gene was detected at both $6 \mathrm{~h}$ and $24 \mathrm{~h}$ by qPCR (Figure 1C). Despite continued presence of NTHi, no impact on MDM viability was detectable, as measured by $\mathrm{LDH}$ release into cell culture supernatants (Figure 1D).

Next, this optimised model was used to visualize NTHi persistence within MDM at $6 \mathrm{~h}$ and $24 \mathrm{~h}$ using a GFP-NTHi strain (GFP-NTHi-375 ${ }^{\mathrm{SR}}$ ). MDM were infected as described and harvested after a gentamicin wash was used to kill and remove all extracellular NTHi. MDM infected with GFP-NTHi had clear evidence of GFP fluorescence at both $6 \mathrm{~h}$ and $24 \mathrm{~h}$, which was closely associated with the macrophage nuclei (Figures 1E-G). The use of a gentamicin wash indicated that the visualized NTHi resided intracellularly within $\mathrm{MDM}$, which was further confirmed by recovery of live GFP-NTHi by viable counting at $6 \mathrm{~h}$ and $24 \mathrm{~h}$ (Figure S2A). Intracellular GFP-NTHi was further quantified by flow cytometry and no significant difference was observed between the two time points (Figures S2B, C).

\section{Distinct Temporal Transcriptomic Profiles Elicited by Macrophages During Intracellular Persistence}

Dual RNASequencing was performed using RNA harvested at the $6 \mathrm{~h}$ and $24 \mathrm{~h}$ time points from five biological repeats. Mapping to the reference human genome accounted for $93 \%$ of total unique reads in uninfected samples (Figure S3). For infected samples, a lower median number of unique reads mapped to the human genome $(77 \%)$, likely due to the presence of NTHi, which accounted for $11 \%$ of the total median unique reads. The remaining reads consisted of unmapped or multiple-mapped reads.

Exploratory analysis of the macrophage data using principal component analysis (PCA) identified two distinct clusters separated by the first principal component (PC1, 69.3\%), which were identified to be either infected or uninfected MDM (Figure 2A). Differential gene expression analysis between uninfected and infected MDM identified 1802 differentially expressed genes (DEGs) at $6 \mathrm{~h}$ and 1763 DEGs at $24 \mathrm{~h}$ (Figure 2B, $\log _{2} \mathrm{FC} \pm 2$, FDR $\mathrm{p}<0.05$ ). This represents $11.9 \%$ and $11.7 \%$ of the starting number of MDM genes (15048) at $6 \mathrm{~h}$ and $24 \mathrm{~h}$, respectively. Overall, a higher number of genes were upregulated ( 1249 at $6 \mathrm{~h}, 1028$ at $24 \mathrm{~h}$ ) compared to downregulated (553 at 6 h, 735 at $24 \mathrm{~h}$ ) in response to NTHi.

To assess regulation of the 1802 and 1763 DEGs at each time point, the $6 \mathrm{~h}$ and $24 \mathrm{~h}$ DEG lists were compared for conserved genes. This comparison identified 863 DEGs (hereafter designated as (core DEGs') to be differentially expressed between uninfected and infected MDM at both $6 \mathrm{~h}$ and $24 \mathrm{~h}$ (Figure 2C). The remaining 1839 DEGs were differentially expressed at a single time point only, with 939 DEGs only 
A

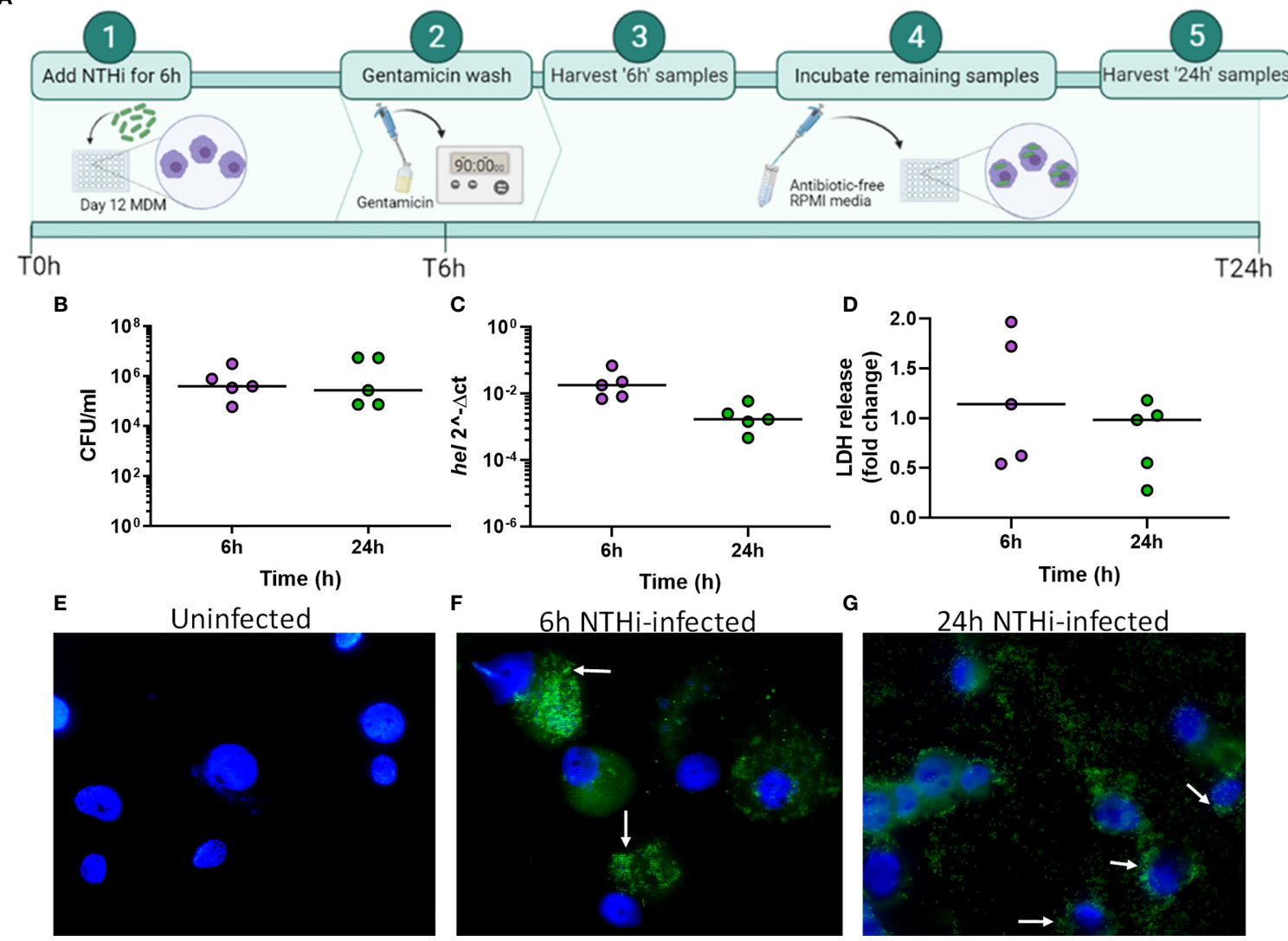

FIGURE 1 | Modelling NTHi infection of macrophages. (A) Model workflow: MDM were challenged with NTHi for $6 \mathrm{~h}$, washed with gentamicin for 90 min to remove extracellular NTHi and left to incubate in antibiotic-free media until $24 \mathrm{~h}$ (created using BioRender.com). (B) The $6 \mathrm{~h}$ and $24 \mathrm{~h}$ time point MDM samples were lysed and plated to quantify the amount of NTHi associated with MDM. (C) RNA was harvested from the $6 \mathrm{~h}$ and $24 \mathrm{~h}$ uninfected and NTHi-infected MDM samples to assess the presence of NTHi RNA through detection of the hel gene by qPCR. Expression of the hel gene was normalised to B2M. (D) MDM viability was not impacted by NTHi ST14 infection, as assessed by LDH release into cell culture supernatants at $6 \mathrm{~h}$ and $24 \mathrm{~h}$. (B-D) $(n=5)$ show paired data and lines indicate medians. Data were analysed by Wilcoxon signed-rank test and no statistical significance was determined. GFP-NTHi was used to visually confirm NTHi association with MDM at the $6 \mathrm{~h}$ and $24 \mathrm{~h}$ time points. Uninfected and NTHi-infected MDM were streaked and fixed onto glass sides followed by staining with DAPI. Slides were visualised using the AxioScope KS400 fluorescence microscope at 100x magnification. (E) Uninfected MDM, (F) GFP-NTHi infected MDM at the $6 \mathrm{~h}$ time point and (G) GFP-NTHi infected MDM at the $24 \mathrm{~h}$ time point. White arrows indicate NTHi associated with MDM cell nuclei.

differentially expressed at $6 \mathrm{~h}$ and 900 DEGs only differentially expressed at $24 \mathrm{~h}$ between uninfected and infected MDM.

The clustering profiles of each DEG group visualised with heatmaps, demonstrated time-dependent expression differences between the three groups. For the $6 \mathrm{~h}$ only DEGs, all $6 \mathrm{~h}$ infected samples clustered together, however they also clustered independently of the $24 \mathrm{~h}$ infected samples (Figure 2D). Similarly, for the $24 \mathrm{~h}$ only DEGs, the $24 \mathrm{~h}$ infected samples clustered together but away from the $6 \mathrm{~h}$ infected samples and the uninfected samples (Figure 2E). In contrast, for the 863 core DEGs, all infected samples clustered independently of the uninfected samples, but clustered together regardless of time point (Figure 2F). The absence of a strong time point signal within this core DEG list was perhaps due to sustained gene expression across time points, emphasised by only 9 out of the 863 genes changing direction of expression between $6 \mathrm{~h}$ and $24 \mathrm{~h}$ (Table S1).
Gene list enrichment analysis found only a small number of significantly enriched GO:terms within the $6 \mathrm{~h}$ only DEG list for all three GO categories. Only 6 terms (1 Biological Process and 5 Cellular Component) were determined to be significantly enriched (FDR p <0.05), with no enrichment in the Molecular Function GO category. In contrast, significantly enriched terms were identified for all three GO categories for the $24 \mathrm{~h}$ only DEGs (Table 1). Finally, significant enrichment of numerous immune response terms in the GO:Biological Process category was identified in the core DEG list (Table 1).

\section{Enrichment of Macrophage Immune Responses During NTHi Persistence}

As the core DEG list was significantly functionally enriched in immune processes, this core DEG list was explored further. To facilitate easier interpretation of functional enrichment, a network of the top 500 significantly enriched Biological 


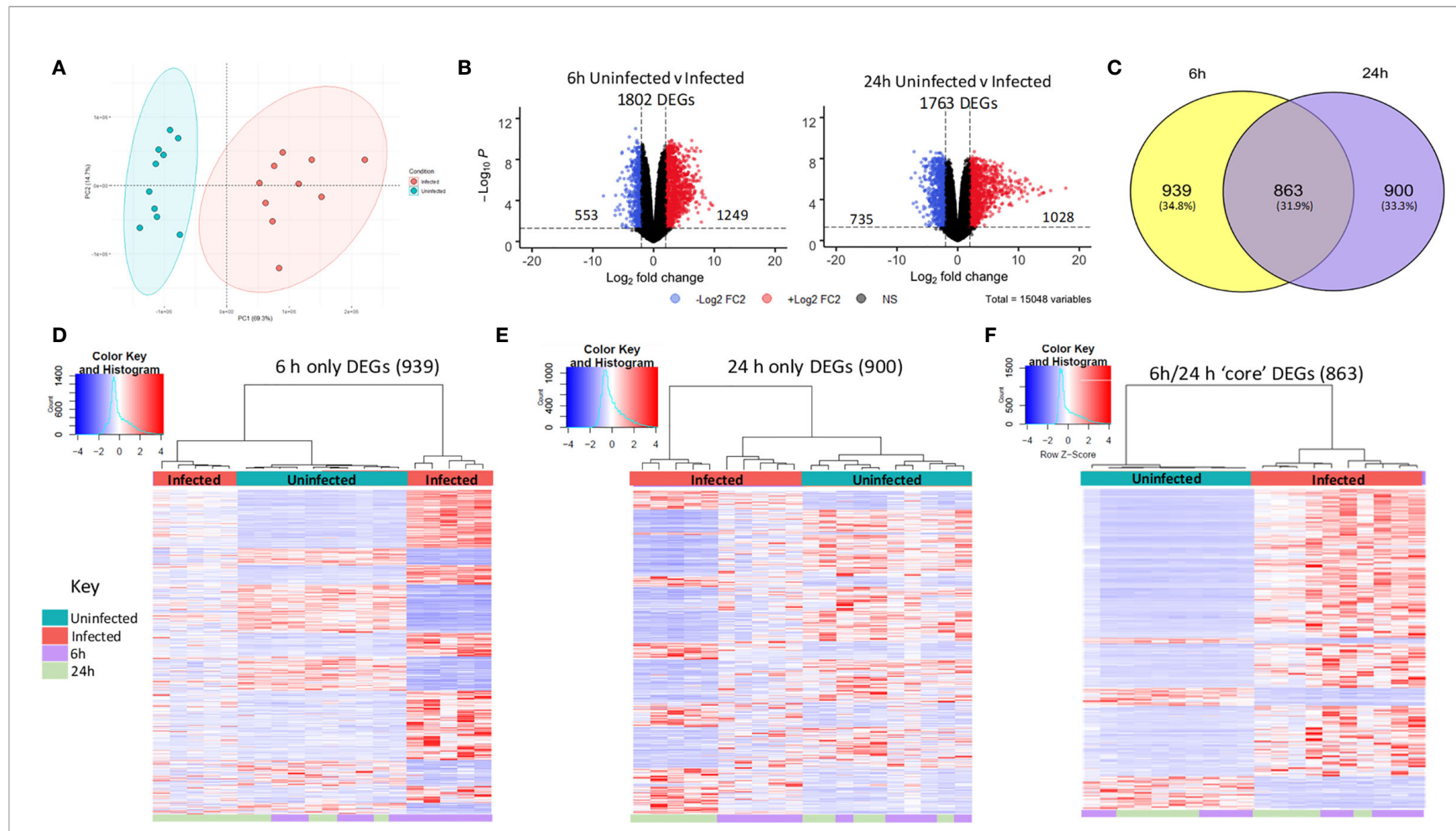

FIGURE 2 | Distinct macrophage transcriptomic profiles in response to NTHi persistence. (A) Principal component analysis of the MDM data set identified that samples clustered based on infection status, with uninfected samples in blue and NTHi infected samples in red. (B) Differential gene expression analysis found 1802 MDM DEGs at the $6 \mathrm{~h}$ time point (left) and 1763 MDM DEGs at $24 \mathrm{~h}$ time point (right) ( $\log _{2}$ FC2 cut off, FDR p $<0.05$ ). (C) Venn diagram showing the regulation of MDM DEGs in a time-dependent manner, with 939 genes only differentially expressed at $6 \mathrm{~h}, 900$ genes only differentially expressed at $24 \mathrm{~h}$ and 863 genes differentially expressed across both $6 \mathrm{~h}$ and $24 \mathrm{~h}$. Heatmap visualisation of the gene expression profiles indicate time-dependent clustering of samples. (D) Clustering of samples based on the expression profile of the MDM DEGs at the $6 \mathrm{~h}$ time point only show clustering of the $6 \mathrm{~h}$ time point sample away from uninfected samples at both time points, as well as the NTHi infected $24 \mathrm{~h}$ time point samples. (E) Similarly, the NTHi infected samples harvested at the $24 \mathrm{~h}$ time point cluster away from all uninfected and $6 \mathrm{~h}$ infected samples. (F) In contrast, based on the expression of the 863 'core' DEGs, the NTHi-infected samples clustered together separately away from the uninfected samples, with no sub clustering based on time point. Clustering was performed using Euclidean distance and Ward linkage methods. Heatmap colour key indicates sample metadata; blue $=$ uninfected samples, red $=$ infected samples, purple $=6 \mathrm{~h}$ time point samples and green $=24 \mathrm{~h}$ time point samples.

Process terms was created using EnrichmentMap (Merico et al., 2010). The network further highlighted the immune response signal in the data, with gene terms clustering together under immune response phrases such as 'immune cell regulation', 'innate response', 'response to bacteria' and 'leukocyte chemotaxis/migration' (Figure 3).

Validation of MDM genes by qPCR confirmed activation of macrophage immune responses. Expression of RELA (p65 subunit for the transcription factor NF- $\mathrm{KB}$ ) and $A C O D 1$ (Aconitate Decarboxylase 1) was significantly increased at both $6 \mathrm{~h}$ (2.4 FC for RELA and $179 \mathrm{FC}$ for ACOD1) and $24 \mathrm{~h}(2.7 \mathrm{FC}$ for RELA and $2759 \mathrm{FC}$ for $A C O D 1)$ compared to the uninfected macrophage controls (all $\mathrm{p}<0.05$, Figures S4A, B).

Protein level validation further demonstrated activation of macrophage pro-inflammatory responses during NTHi persistence, with increased levels of IL-1 $\beta$, IL- 6 , TNF- $\alpha$, and IL-10 released into culture supernatants detected in all infected samples compared to uninfected samples at each time point (all $\mathrm{p}<0.05$, Figures S4C-F). Increased MDM release of neutrophil (IL-8, IL-17C) and lymphocyte (IL-15, IL-36 $\beta$ ) related mediators in cell culture supernatants was also observed (Figures S4G-J).

\section{Enrichment of Macrophage Intracellular Immune Response Pathways Indicates Intracellular Residence of NTHi}

A total of 75 KEGG pathways were significantly functionally enriched [ShinyGo (Ge et al., 2020), FDR p <0.05, hypergeometric test] in the core DEG list, which included a number of immune response pathways (Figure 4A). Significant enrichment of specific intracellular immune response pathways such as the 'NOD-like receptor signalling', 'Influenza A' and 'Cytosolic DNA-sensing' KEGG pathways indicate activation of macrophage intracellular responses during NTHi persistence.

Within the Cellular Component category, enrichment of the GO:term 'symbiont-containing vacuole' (GO:0020003, FDR $\mathrm{p}=0.0433$, Figure 4B), also suggest intracellular responses to NTHi. The genes enriched in this category (GBP2, GBP4, GBP6, and $G B P 7$ ) are members of the guanylate-binding protein (GBP) family, which play a role in antibacterial defence against intracellular pathogens. Although not included within this particular GO:term annotation, 3 other GBP family members (GBP1, GBP3 and GBP5) were also differentially expressed at both $6 \mathrm{~h}$ and $24 \mathrm{~h}$ (all FDR $\mathrm{p}<0.05$, Figure 4C). 
TABLE 1 | Time-dependent enrichment of macrophage processes during NTHi persistence.

\begin{tabular}{|c|c|c|c|c|c|}
\hline Category & GO:ID & GO:Term & Enrichment FDR & Genes in input & Genes in annotation \\
\hline \multicolumn{6}{|l|}{ 6h only DEGs } \\
\hline GO: Biological Process & GO:0001816 & cytokine production & 0.05 & 71 & 875 \\
\hline \multirow[t]{5}{*}{ GO: Cellular Component } & GO:0098589 & membrane region & 0.03 & 39 & 411 \\
\hline & GO:0045121 & membrane raft & 0.03 & 37 & 396 \\
\hline & GO:0098857 & membrane microdomain & 0.03 & 37 & 397 \\
\hline & GO:0016324 & apical plasma membrane & 0.04 & 35 & 373 \\
\hline & GO:0000790 & nuclear chromatin & 0.05 & 121 & 1923 \\
\hline $\begin{array}{l}\text { GO: Molecular Function } \\
24 \mathrm{~h} \text { only DEGs }\end{array}$ & \multicolumn{4}{|c|}{$24 \mathrm{~h}$ only DEGs } & NA \\
\hline \multirow[t]{5}{*}{ GO: Biological Process } & GO:0022610 & biological adhesion & 1.93E-05 & 116 & 1516 \\
\hline & GO:0042493 & response to drug & 1.93E-05 & 97 & 1194 \\
\hline & GO:0007155 & cell adhesion & 1.93E-05 & 115 & 1509 \\
\hline & GO:0046903 & secretion & 2.88E-05 & 132 & 1835 \\
\hline & GO:0006952 & defense response & 3.85E-05 & 132 & 1850 \\
\hline \multirow[t]{5}{*}{ GO: Cellular Component } & GO:0031226 & intrinsic component of plasma membrane & 4.87E-17 & 161 & 1790 \\
\hline & GO:0005887 & integral component of plasma membrane & $4.87 \mathrm{E}-17$ & 156 & 1710 \\
\hline & GO:0031012 & extracellular matrix & $1.21 \mathrm{E}-04$ & 55 & 598 \\
\hline & GO:0098797 & plasma membrane protein complex & 1.83E-04 & 61 & 711 \\
\hline & GO:0062023 & collagen-containing extracellular matrix & $1.83 E-04$ & 46 & 474 \\
\hline \multirow[t]{5}{*}{ GO: Molecular Function } & GO:0005178 & integrin binding & 1.36E-03 & 23 & 146 \\
\hline & GO:0015267 & channel activity & 1.36E-03 & 62 & 705 \\
\hline & GO:0022803 & passive transmembrane transporter activity & 1.36E-03 & 62 & 706 \\
\hline & GO:0022836 & gated channel activity & 1.36E-03 & 48 & 491 \\
\hline & GO:0022843 & voltage-gated cation channel activity & 1.73E-03 & 30 & 245 \\
\hline \multicolumn{6}{|l|}{ 6h24h 'core' DEGs } \\
\hline \multirow[t]{5}{*}{ GO: Biological Process } & GO:0006952 & defense response & $4.82 E-51$ & 228 & 1850 \\
\hline & GO:0034097 & response to cytokine & $1.51 \mathrm{E}-48$ & 183 & 1287 \\
\hline & GO:0043207 & response to external biotic stimulus & 1.17E-44 & 200 & 1606 \\
\hline & GO:0051707 & response to other organism & $2.36 E-44$ & 199 & 1604 \\
\hline & GO:0019221 & cytokine-mediated signaling pathway & $2.36 \mathrm{E}-44$ & 138 & 814 \\
\hline \multirow[t]{5}{*}{ GO: Cellular Component } & GO:0005887 & integral component of plasma membrane & 5.91E-07 & 123 & 1710 \\
\hline & GO:0031226 & intrinsic component of plasma membrane & 5.91E-07 & 127 & 1790 \\
\hline & GO:0009986 & cell surface & $1.51 \mathrm{E}-06$ & 84 & 1036 \\
\hline & GO:0031012 & extracellular matrix & 6.59E-03 & 48 & 598 \\
\hline & GO:0065010 & extracellular membrane-bounded organelle & 1.09E-02 & 5 & 9 \\
\hline \multirow[t]{5}{*}{ GO: Molecular Function } & GO:0005125 & cytokine activity & $3.92 E-32$ & 65 & 225 \\
\hline & GO:0048018 & receptor ligand activity & 7.37E-23 & 82 & 497 \\
\hline & GO:0005126 & cytokine receptor binding & $7.40 E-23$ & 64 & 312 \\
\hline & GO:0030546 & signaling receptor activator activity & $7.40 \mathrm{E}-23$ & 82 & 502 \\
\hline & GO:0030545 & receptor regulator activity & $2.10 E-20$ & 82 & 547 \\
\hline
\end{tabular}

A maximum of 5 of the most significantly functionally enriched terms for each category are shown, with fewer terms meaning lower enrichment significance for a specific category or time point. Genes in input show the number of MDM genes assigned to each term, which were compared against the full gene list for each category used by ToppFunn/ToppGene.

\section{NTHi Transcriptomic Regulation During Adaptation to Intracellular Persistence}

Despite activation of macrophage innate immune responses, NTHi was still able to persist within MDM for the duration of this model. Thus, the NTHi transcriptome was assessed to determine NTHi transcriptomic adaptations to intracellular persistence. PCA identified two distinct clusters separated by the first principal component (94.3\%) which was associated with the $6 \mathrm{~h}$ or $24 \mathrm{~h}$ time point (Figure 5A). Differential gene expression analysis identified 107 DEGs between $6 \mathrm{~h}$ and $24 \mathrm{~h}$, with 69 upregulated and 38 downregulated DEGs (Figure 5B, $\log _{2} \mathrm{FC} \pm 1$, FDR p <0.05).

\section{NTHi Modulation of Metabolic Pathways During Intracellular Persistence}

Gene list enrichment analysis of the NTHi DEG list identified numerous significantly enriched terms in the Biological Process,
Molecular Function and Cellular Component categories (Figure S5). Clustering the significantly enriched Biological Process terms identified that the majority of significantly enriched GO:term clusters were metabolic related, with 'vitamin biosynthesis', 'amino acid metabolism', 'nucleoside metabolism' and 'fatty acid synthesis' clusters identified (Figure 5C). Due to the high redundancy and ambiguity surrounding gene ontology terms, the functional role of the 107 DEGs was summarised using the results of the enrichment analysis and gene function (Figure 6A). The highest number of genes (29) were primarily involved in metabolic processes. The remaining genes were involved in regulation of gene expression (23), stress responses (8), virulence (5), replication (5) and protein regulation (2). The 35 remaining genes were uncharacterised, resulting in no gene name or function being available for analysis. Of these uncharacterised genes, 22 were hypothetical protein coding genes, 9 were transcripts assigned as novel genes and 2 were sRNA. 


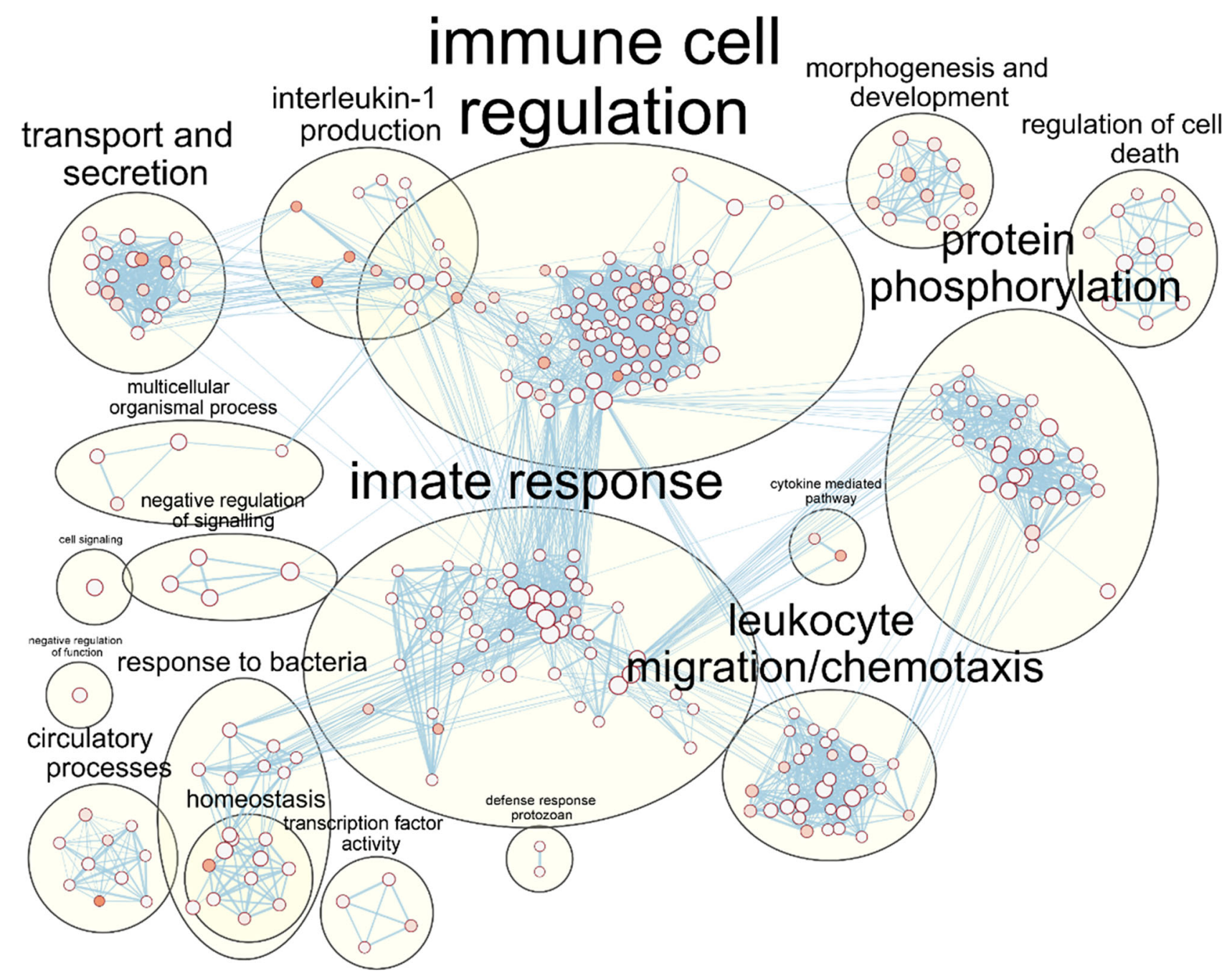

FIGURE 3 | Enrichment of macrophage immune responses during NTHi persistence. Enrichment analysis using ToppFunn identified over 500 significantly enriched biological processes which were clustered using EnrichmentMap and AutoAnnotate in Cytoscape to identify the key biological processes involved in the MDM response to NTHi. Nodes represent individual GO:terms, with size relating to the number of genes in each term and the colour indicating enrichment significance. Edges represent connections between nodes that share genes.

Out of the 107 DEGs, 29 metabolic-associated genes were identified, with upregulation of 5 genes $(r s x C, r s x D, r s x E, r s x G$ and $c y d D$ ) involved in aerobic respiration. The remaining genes, except $y$ hje_ 1 , were assigned to an alternative metabolic pathway, revealing a diverse array of metabolic pathways including biotin (vitamin B7), riboflavin (vitamin B2) and thiamine (vitamin B1) pathways (Figure 6B). Other annotated clusters demonstrated enrichment of bacterial stress responses ('stress/stimulus response' and 'response to toxic substance' clusters) and processes involved in gene expression and protein synthesis ('ribosome biogenesis' and 'tRNA/ncRNA processing' clusters).

Although KEGG pathway analysis confirmed enrichment of several metabolic KEGG pathways, the most significantly enriched KEGG pathway was the 'Ribosome' pathway (Figure 6C). All 11 DEGs assigned to this pathway were downregulated at $24 \mathrm{~h}$. In total,
46 ribosomal protein genes were present in the annotated NTHi gene list, with 37 genes downregulated at $24 \mathrm{~h}$ (all FDR $\mathrm{p}<0.05$, Figure 6D). However, only 11 of these 37 DEGs $(r p l B, r p l C, r p l D$, $r p l L, r p l P, r p l V, r p l W, r p m C$, rpsI, $r p s J$ and $r p s Q$ ) were above the $\log _{2}$ FC1 cut off. Nonetheless, it was clear that between $6 \mathrm{~h}$ and $24 \mathrm{~h}$, NTHi globally downregulated the expression of ribosomal protein genes.

\section{The Top Regulated NTHi Genes During Intracellular Persistence Were Differentially Expressed Compared to Planktonic NTHi}

It was important to confirm that NTHi DEGs were only differentially expressed during intracellular persistence of MDM. Two significantly upregulated DEGs ( $b i o C$ and $m e p M$ ) and the top 
A

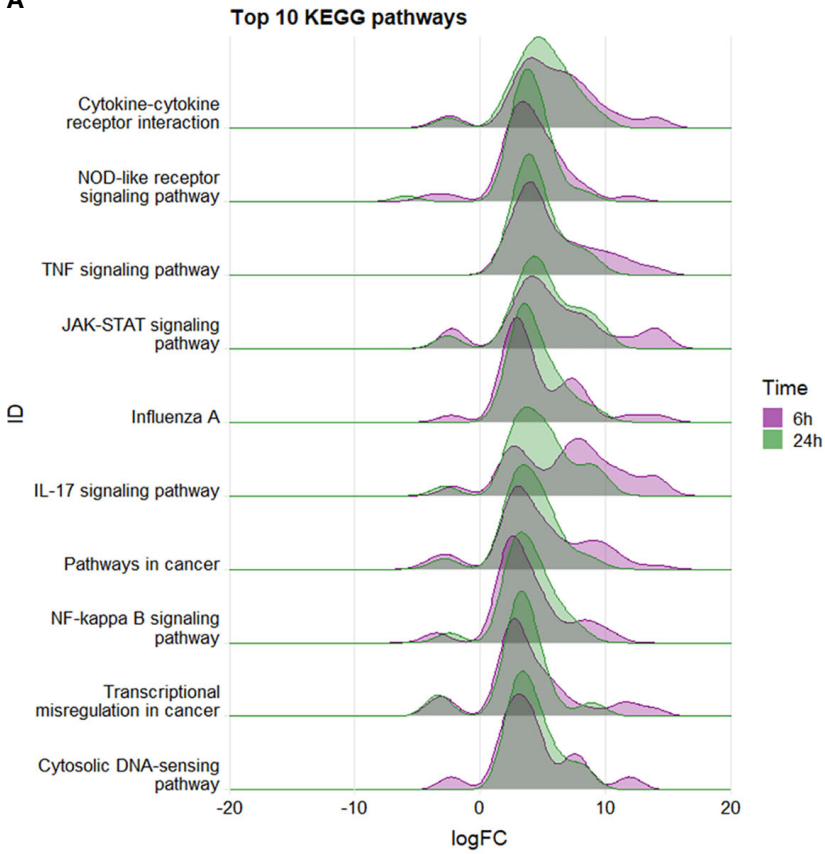

\begin{tabular}{|c|c|c|c|}
\hline ID & Term & p-value & $\mathbf{I} / \mathbf{T}$ \\
\hline \multicolumn{4}{|c|}{ O: Biological Processes } \\
\hline $0: 0043903^{r}$ & $\begin{array}{c}\text { gulation of symbiosis, encompassing mutualism } \\
\text { through parasitism }\end{array}$ & $1.35 \mathrm{E}-10$ & $38 / 232$ \\
\hline $0: 0044403$ & symbiotic process & $3.57 \mathrm{E}-04$ & $67 / 911$ \\
\hline $0: 0051817^{n}$ & $\begin{array}{l}\text { dification of morphology or physiology of other } \\
\text { organism involved in sym biotic interaction }\end{array}$ & 1.57E-02 & $15 / 124$ \\
\hline \multicolumn{4}{|c|}{ O:Cellular Component } \\
\hline $0: 0020005$ & symbiont-containing vacuole membrane & $2.47 \mathrm{E}-02$ & $4 / 6$ \\
\hline $0: 0020003$ & symbiont-containing vacuole & 4.33E-02 & $4 / 7$ \\
\hline
\end{tabular}

C

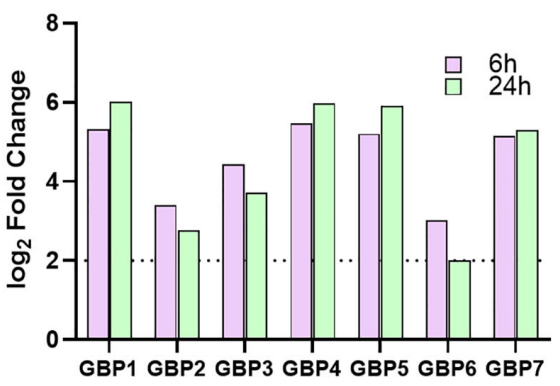

FIGURE 4 | Enrichment of macrophage intracellular immune responses during NTHi persistence. (A) KEGG pathway analysis identified a number of enriched immune processes, with a number of pathways indicating a response to an intracellular pathogen. The genes assigned to each process were more highly upregulated at both $6 \mathrm{~h}$ (purple) and $24 \mathrm{~h}$ (green). Pathway/category IDs are ordered by enrichment significance (FDR). (B) Significantly enriched GO terms in the Biological Process and Cellular Component categories relating to host-pathogen symbiosis for the 863 core genes differentially expressed at $6 \mathrm{~h}$ and $24 \mathrm{~h}$ (log $2 \mathrm{FC} \pm$ 2, FDR $p<0.05$ ). P-value indicates the enrichment FDR, I = input number of genes, $\mathrm{T}=$ total number of genes in annotation. (C) MDM upregulation of guanylatebinding proteins (GBPs) 1-7 involved in host response to intracellular pathogens. Purple bar $=6 \mathrm{~h}$, green bar $=24 \mathrm{~h}$. Dotted line indicates log 2 FC2 cut off. All genes were statistically significantly upregulated at both time points (FDR $p<0.05$ ).

significantly downregulated DEG (dps) were selected for in vitro investigations. The expression of bioC (1.8 FC, $\mathrm{p}=0.0156)$, mepM (2.5 FC, $\mathrm{p}=0.0313)$ and $d p s(0.4 \mathrm{FC}, \mathrm{p}=0.0156)$ during NTHi persistence in MDM was first validated by qPCR (Table S2).

Next, the expression of each gene was compared between planktonic NTHi and intracellular NTHi at $6 \mathrm{~h}$ and $24 \mathrm{~h}$. All three genes were more highly expressed by intracellular NTHi. The two upregulated DEGs bioC and mep $M$ were more highly expressed at $24 \mathrm{~h}(\mathrm{p}=0.0014$ and $\mathrm{p}=0.0178$, respectively, Figures 7A, B). The $d p s$ gene was more highly expressed by NTHi at $6 \mathrm{~h}$ compared to planktonic ( $\mathrm{p}=0.0018)$, with expression levels decreasing by $24 \mathrm{~h}$ $(\mathrm{p}=0.0954)$, in line with the decrease in expression detected by dual RNASeq and qPCR validation between $6 \mathrm{~h}$ and $24 \mathrm{~h}$ (Figure 7C). Despite this decrease in expression, $d p s$ expression levels did not revert back to similar expression levels as planktonic NTHi, indicating the regulation of these genes during intracellular persistence were distinct compared to planktonic NTHi.

\section{Strain-Dependent Differences in NTHi Transcriptomic Adaptations During Intracellular Persistence}

As NTHi strains are heterogeneous and have been suggested to have different capacities to persist within host cells (Craig et al., 2001), we also assessed whether the expression of the top three identified
NTHi genes were conserved in additional clinical strains during intracellular persistence of MDM at $24 \mathrm{~h}$. The genomic relatedness of seven clinical strains of NTHi isolated from nasal brushes, lung protected brushes or sputum obtained from patients with chronic respiratory disease was assessed by ParSNP (Treangen et al., 2014) (Figure 8A). Three strains were selected based on the diversity inferred from the phylogenetic tree, including the ST14 strain, which has been used for this work so far. To ensure diverse strains were selected, one strain from each clade representing a different anatomical sampling location was chosen: ST408 (nasal brushing), ST14 (lung protected brushing), ST201 (sputum).

All three strains were able to persist within MDM (Figure 8B), however higher levels of ST201 were recovered from MDM at $6 \mathrm{~h}$ ( $\mathrm{p}=0.0016$ compared to ST408) and $24 \mathrm{~h}(\mathrm{p}=0.0281$ compared to both ST14 and ST408). When comparing the expression of each of the three genes across strains, it was apparent that NTHi ST201 modulated expression of the three genes more robustly than ST14 and ST408 during persistence (Figure 8C).

\section{DISCUSSION}

Rapid advances in sequencing technologies now allow for simultaneous profiling of host-pathogen interactions, giving 


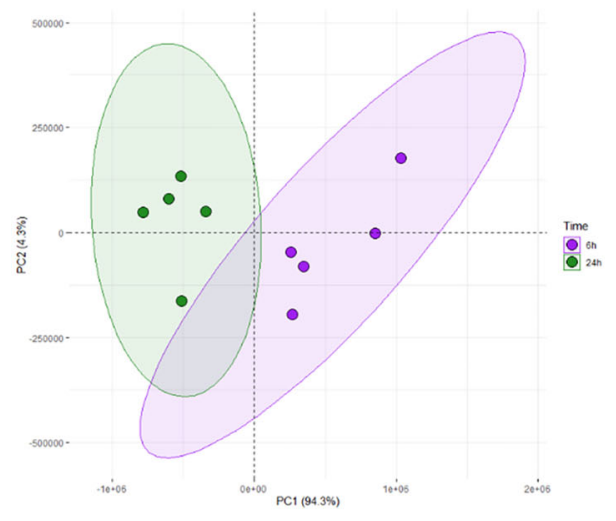

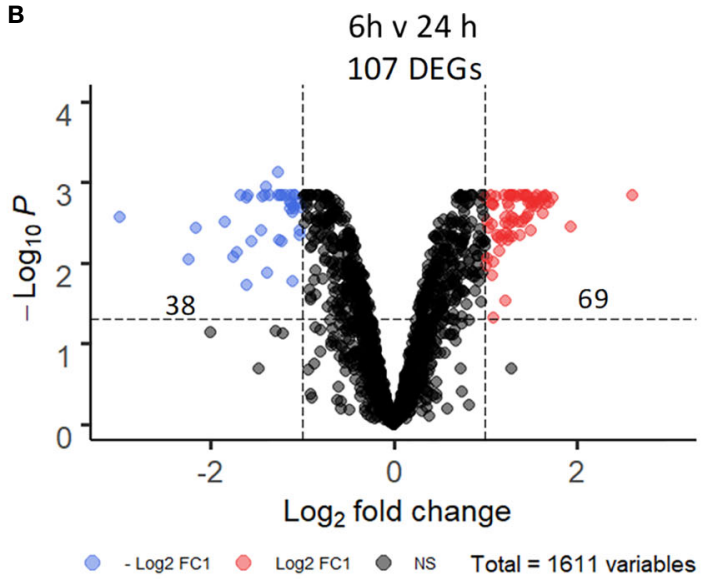

C

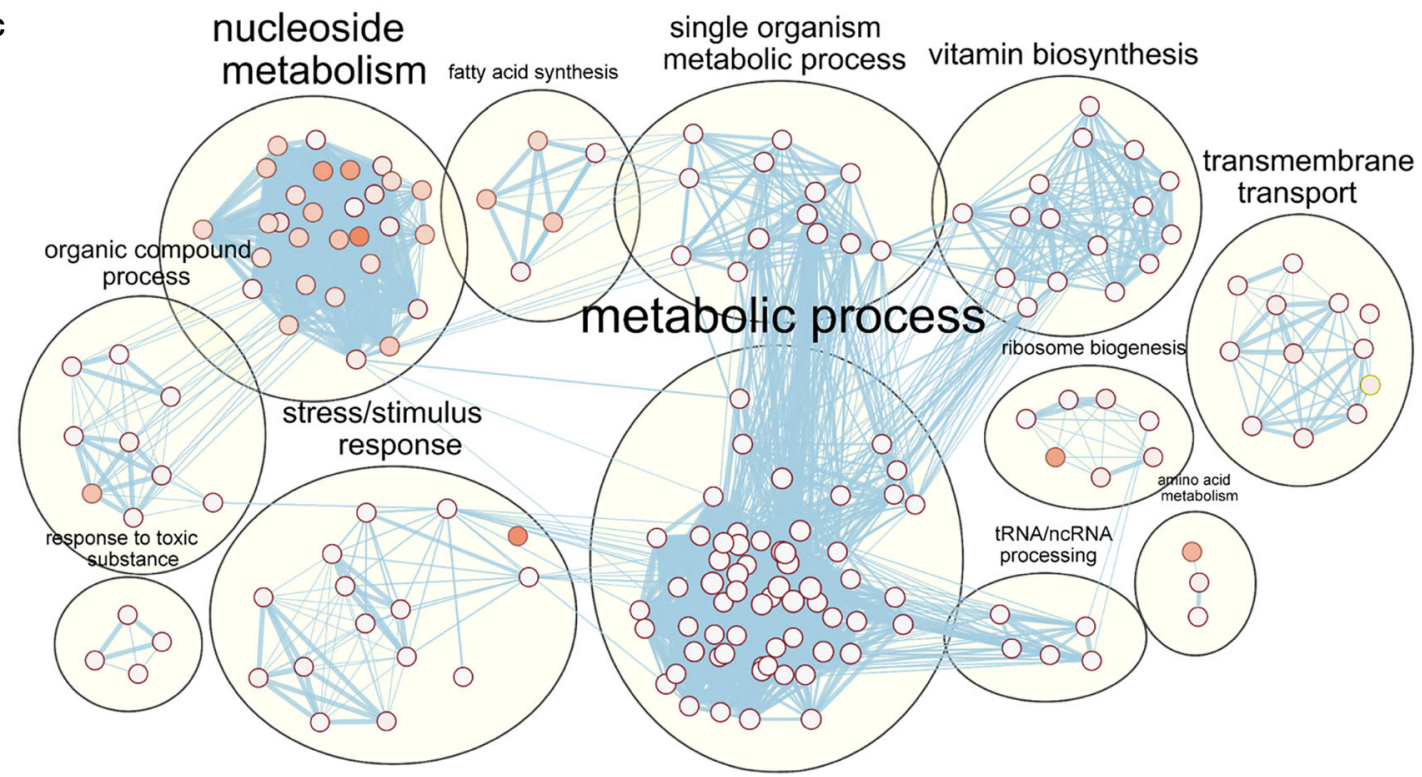

FIGURE 5 | NTHi transcriptomic regulation during adaptation to intracellular persistence. (A) Principal component analysis identified clustering of NTHi samples based on time point (6 $\mathrm{h}$ time point samples in purple and $24 \mathrm{~h}$ time point samples in green). (B) Differential gene expression analysis identified $107 \mathrm{NTHi}$ DEGs at $24 \mathrm{~h}$ ( $\log _{2}$ FC1 cut off, FDR $\mathrm{p}<0.05$ ). (C) Clustering of the enriched Biological Process GO:terms performed using EnrichmentMap and AutoAnnotate in Cytoscape found enrichment of numerous metabolic processes. Nodes represent individual GO:terms, with size relating to the number of genes in each term and the colour indicating enrichment significance. Edges represent connections between nodes that share genes.

novel insights into the cellular cross-talk occurring during clinically relevant infections. NTHi is still considered an extracellular pathogen, despite increasing evidence of an intracellular lifestyle (Craig et al., 2001; Morey et al., 2011; Olszewska-Sosińska et al., 2016). NTHi was first demonstrated to be residing and replicating within the phagocytic compartment of mononuclear cells (Farley et al., 1986), but in epithelial cells, NTHi colocalised with acidic compartments displaying late endosomal features and did not appear to be replicating (Morey et al., 2011). Subsequent in vitro studies have shown the ability of NTHi to invade and persist within monocytes and macrophages (Ahrén et al., 2001; Craig et al., 2001, 2002; King et al., 2008). The mechanism underlying this NTHi persistence within host cells is unclear. As such, we used dual RNASeq to simultaneously assess host and pathogen transcriptomic changes during intracellular persistence to better understand the mechanism of NTHi persistence within human macrophages.

Analysis of macrophage transcriptomic changes in response to NTHi persistence identified time-dependent macrophage responses. Specifically, a core transcriptomic profile was consistently expressed across both $6 \mathrm{~h}$ and $24 \mathrm{~h}$, which was enriched in intracellular immune responses. A component of the macrophage intracellular pathogen detection machinery includes GBPs, of which all 7 GBPs were upregulated during NTHi persistence. The importance of GBPs in restricting intracellular pathogens have been shown in studies using GBP-deficient macrophages, which demonstrate impaired responses 
A

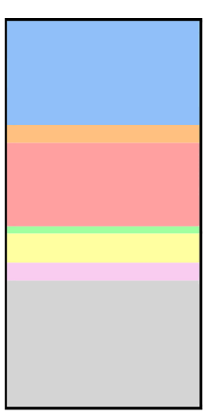

$\square$ Metabolic processes

$\square$ Replication

$\square$ Regulation of gene expression

$\square$ Protein regulation

$\square$ Stress response

$\square$ Virulence

$\square$ Uncharacterised

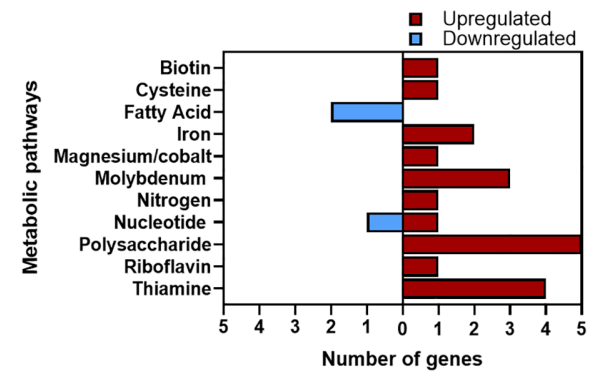

Total $=107$

C

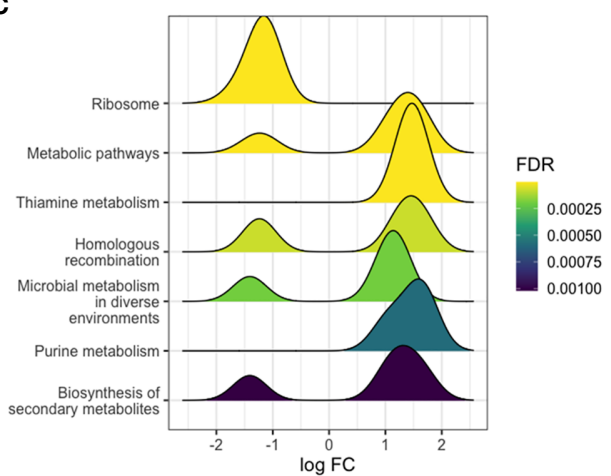

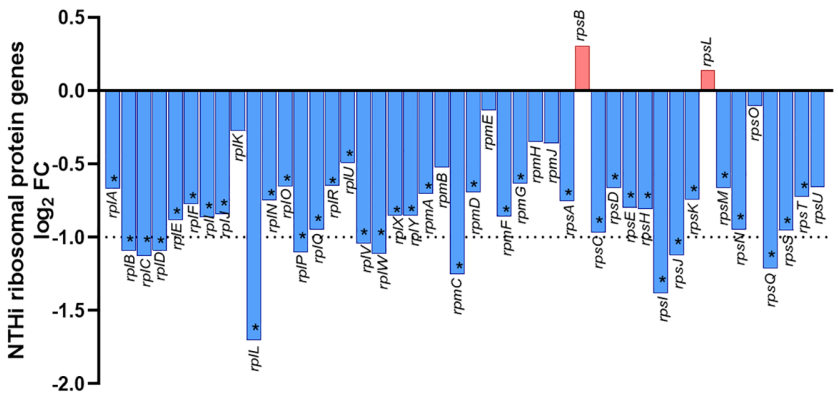

FIGURE 6 | Modulation of NTHi processes during adaption to intracellular persistence. (A) The stacked bar chart highlights the main processes that the 108 NTHi DEGs are involved in. The process with the highest number of genes was metabolic processes (29), followed by regulation of gene expression (23), stress responses (8), virulence (5), replication (5) and protein regulation (2). The remaining genes (36) were uncharacterised (hypothetical, novel genes or sRNA). (B) Genes involved in bacterial metabolism were assigned to specific alternate metabolic pathways. Red = upregulated, blue = downregulated. (C) KEGG pathway analysis identified enrichment of KEGG pathways during intracellular persistence, ordered by enrichment significance (FDR). (D) KEGG pathway analysis identified the most significantly enriched pathway was 'Ribosome'. Exploration of ribosomal protein gene expression identified global downregulation of NTHi ribosomal protein genes during infection. Bar chart shows $\log _{2}$ FC values of the 46 ribosomal protein genes detected in the NTHi data set. Dotted line indicates $\log _{2}$ FC1 cut off, with asterisk indicating genes (37) that were determined to be significantly differentially expressed at FDR $p<0.05$.

A

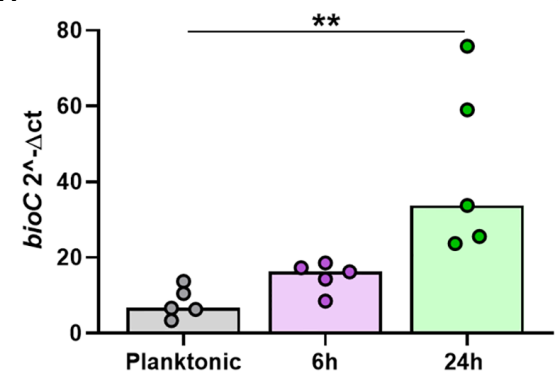

B

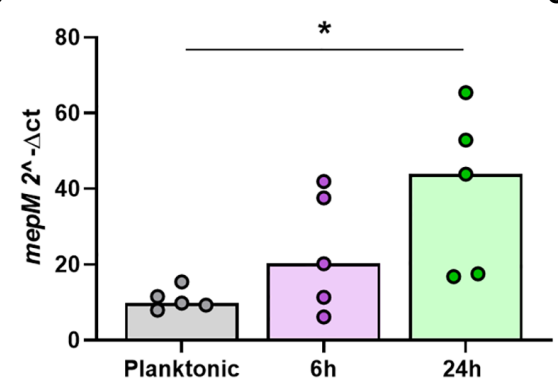

C

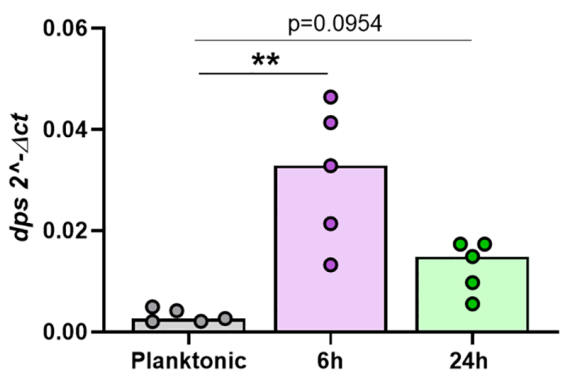

FIGURE 7 | The top regulated NTHi genes during intracellular persistence were differentially expressed compared to planktonic NTHi. To compare gene expression between planktonic and intracellular, persisting NTHi, RNA was harvested from NTHi ST14 grown in culture media alone (regarded as planktonic NTHi) or from NTHiinfected MDM at the $6 \mathrm{~h}$ and $24 \mathrm{~h}$ time points, as previously described $(\mathrm{n}=5)$. The expression of the top regulated NTHi genes (A) bioC, (B) mepM and (C) $d p s$ was assessed by qPCR. Gene expression was normalised to NTHi rho gene. Graphs show unpaired data and lines indicate medians. $N=5$. Data were analysed using a Kruskal-Wallis test with Dunn's multiple comparisons; ${ }^{*} \mathrm{p}<0.05,{ }^{* *} \mathrm{p}<0.01$. 
A

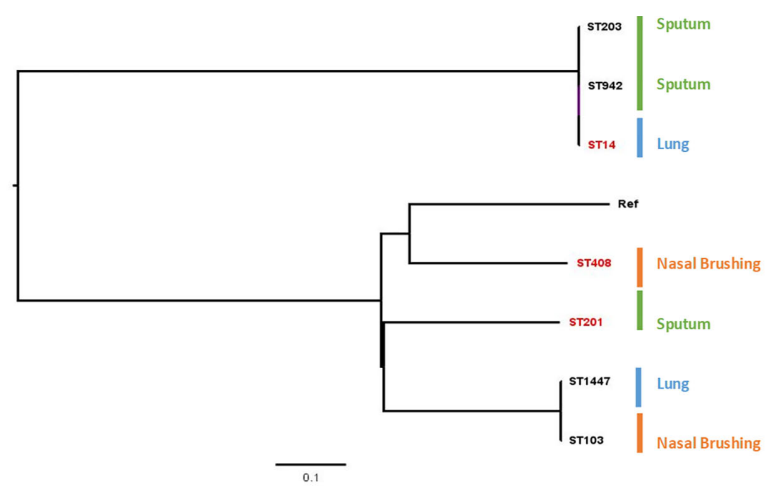

B

$6 \mathrm{~h}$
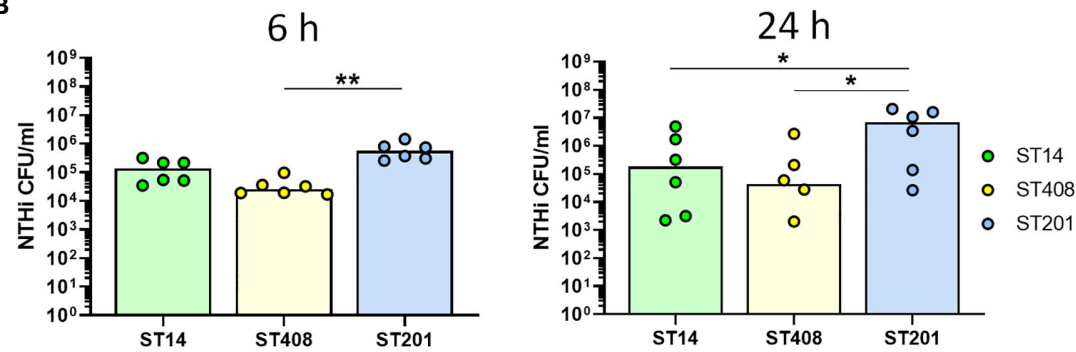

C
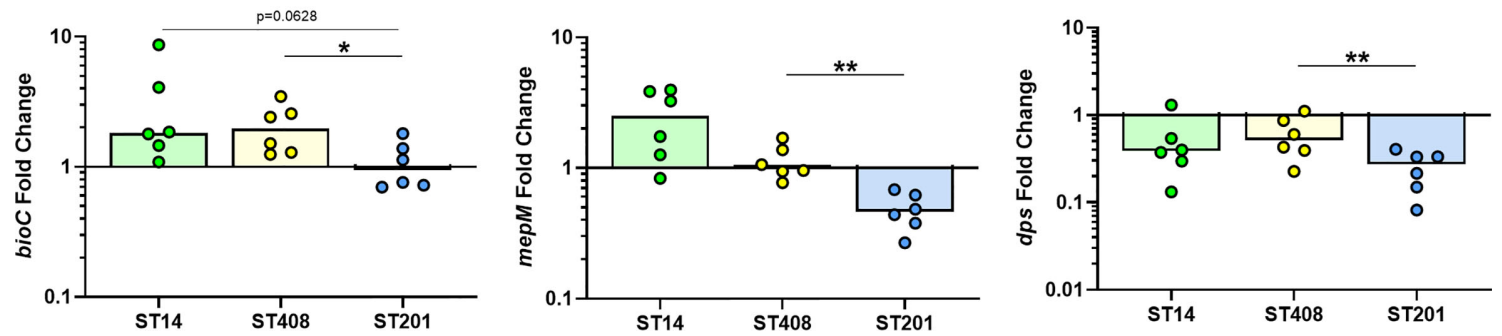

FIGURE 8 | Strain-dependent differences during NTHi persistence. (A) The diversity of seven clinical NTHi isolates were assessed by ParSNP using default parameters and NTHi 86-028NP as the reference strain. Strains were isolated from either from sputum sample (green), nasal brushing (orange) or protected bronchial brushes of the lung (blue). Phylogenetic tree was created in FigTree using ParSNP output files and strains highlighted in red (ST14, ST408 and ST201) indicate the strains chosen for further in vitro experimental analysis. (B) Invasion and persistence within MDM by the three chosen different strains of NTHi was measured by live viable counting at the $6 \mathrm{~h}$ and $24 \mathrm{~h}$ time points. (C) Expression of the top regulated NTHi genes were differentially expressed by additional clinical strains of NTHi during intracellular persistence. Gene expression was normalised to NTHi rho gene and data are shown as fold change in expression from $6 \mathrm{~h}$ to $24 \mathrm{~h}$ Graphs show paired data and lines indicate medians. $N=6$. Data were analysed using Friedman test with Dunn's multiple comparisons; ${ }^{*} p<0.05,{ }^{* *} p<0.01$.

to intracellular pathogens including Mycobacterium bovis, L. monocytogenes, Francisella novis and Salmonella typhimurium (Kim et al., 2011; Meunier et al., 2014; Meunier et al., 2015). GBPs are thought to facilitate rupturing of either bacteria-containing vacuoles or bacteria present in the cytosol. Subsequent release of bacterial content results in cytosolic detection of the invading pathogen and inflammasome activation (Meunier et al., 2014; Meunier et al., 2015), which could explain macrophage enrichment of the 'Cytosolic DNA-sensing' pathway in response to NTHi.

Conversely, recent work has shown GBPs associate with the bacterial surface moments after bacterial escape from host vacuoles, without bacteriolysis (Santos et al., 2020). The second highest upregulated NTHi DEG was an endopeptidase, mерM, which could suggest GBP-NTHi interactions. Endopeptidases are responsible for incorporation of peptidoglycan into the bacterial cell wall, a crucial process not just for bacterial growth and replication, but also bacterial cell viability. Thus, upregulation of mepM could suggest activation of NTHi defences against host immune mechanisms that target the cell wall of bacteria. Functional work investigating physical NTHi-GBP interactions within MDM are therefore warranted to assess the role of GBPs in the response to intracellular NTHi.

We identified $A C O D 1$ as one of the top most significantly upregulated MDM genes across both $6 \mathrm{~h}$ and $24 \mathrm{~h}$. ACOD1 encodes for a cis-aconitate decarboxylase involved in the production of itaconate from cis-aconitate produced in the TCA (Kreb) cycle (Michelucci et al., 2013). Itaconate inhibits succinate dehydrogenase ( $\mathrm{SDH})$, resulting in accumulation of succinate and diversion of macrophage metabolism towards aerobic glycolysis (O'Neill and Artyomov, 2019). ACOD1 was previously designated IRG1 (immune responsive gene 1) as it 
appeared to play an unknown function in the inflammatory immune response, with increased gene expression measured in LPS-stimulated macrophages (Lee et al., 1995). ACOD1 and itaconate are suggested to be immunomodulatory, with studies demonstrating that $A C O D 1$ expression was important for the host immune response to Mycobacterium tuberculosis (Mtb) infection (Nair et al., 2018; Hoffmann et al., 2019). However, Michelucci et al., confirmed that itaconate also functions as an antimicrobial metabolite, important for restricting growth of Mtb and Salmonella enterica (Michelucci et al., 2013).

In contrast, some pathogens can utilise itaconate to enhance pathogenicity. Riquelme et al. found that in response to itaconate, Pseudomonas aeruginosa adapted metabolic activity towards biofilm formation and extracellular polysaccharide (EPS) production, which in turn resulted in increased itaconate release from host cells (Riquelme et al., 2020). Similarly, Salmonella has shown to be able to sense and respond to macrophage itaconate by upregulating expression of itaconate degradation proteins (Hersch and Navarre, 2020). It is not known whether NTHi is similarly able to interfere with ACOD1/ itaconate regulation of macrophage inflammatory processes, however the presence of genes for itaconate degradation in numerous other bacteria suggests this possibility (Sasikaran et al., 2014). Further work assessing the exact impact of the antibacterial and immunomodulatory activity of itaconate during NTHi persistence will help identify whether these properties of itaconate can feasibly be used therapeutically to reduce the burden of NTHi persistence in chronic respiratory diseases. This is particularly important given recent findings that airway itaconate levels and expression levels of ACOD1 were decreased in AM from patients with IPF compared to controls (Ogger et al., 2020).

A crucial macrophage function is to orchestrate and regulate the immune response, which includes recruitment and activation of other immune cells (Arango Duque and Descoteaux, 2014). NTHi persistence has been specifically associated with a switch to T17 and neutrophilic inflammation in asthma (Yang et al., 2018). In this current work, KEGG analysis identified enrichment of IL-17 signalling, with upregulation of numerous macrophage genes for neutrophil and $\mathrm{T}$ cell chemoattractants present in the 'leukocyte chemotaxis/migration' biological process cluster. Macrophage release of specific neutrophilassociated mediators (IL-8 and IL-17C) was detected in cell culture supernatants. NTHi-infected macrophages could be a cellular source of neutrophil chemoattractants in vivo, driving the recruitment of neutrophils to the lung. This has previously been postulated by Song et al., who suggested that alveolar macrophages are the cellular source of increased IL-17 in the BALF of asthmatic patients, not Th17 cells (Song et al., 2008).

In contrast, Singhania et al., suggested that activated $\mathrm{T}$ cells drive an IL-17 response in severe asthma (Singhania et al., 2018). We have previously demonstrated the ability of macrophages to activate $\mathrm{T}$ cells in response to challenge with influenza A (Staples et al., 2015) or NTHi (Wallington et al., 2018). In particular, a role for the PD1/PDL1 exhaustion pathway in regulating $\mathrm{T}$ cell function has been suggested to contribute to dysfunctional cytotoxic responses and impaired immune regulation in an experimental lung explant model (McKendry et al., 2016). Given the vital role of the macrophage in immune regulation, the complex, inflammatory environment in asthma may be driven by dysregulation of the NTHi-infected macrophages ability to recruit and activate both neutrophils and $\mathrm{T}$ cells.

Intracellular invasion and persistence within host cells is a strategy employed by numerous bacteria to evade the immune response. As described previously, invasion of macrophages by NTHi has been reported by numerous studies (Ahrén et al., 2001; Craig et al., 2001; Craig et al., 2002; King et al., 2008; Morey et al., 2011), but the mechanism of persistence and survival of NTHi within macrophages is unclear. Following invasion of host cells, bacteria are faced with a hostile environment which is only available as a niche for those bacteria able to adapt to unfavourable conditions. Macrophage activation of responses are crucial for killing intracellular pathogens, with some pathogens such as Mtb able to persist within macrophages that have become activated, whereas other pathogens, such as Listeria monocytogenes are more readily killed following activation of macrophage intracellular immune responses (Kaufmann and Dorhoi, 2016). Thus, bacterial adaptations to a hostile intracellular environment can determine the ability of pathogens to persist. Adaptations to environmental changes, such as nutrient or oxygen availability, can be regulated by the bacterial stringent response (Wilson and Nierhaus, 2007). Differential regulation of NTHi genes involved in various metabolic pathways, stress responses and the ribosome pathway, as well as no change in NTHi CFU between $6 \mathrm{~h}$ and $24 \mathrm{~h}$, suggested potential activation of the bacterial stringent response by NTHi to enhance intracellular survival.

Global modulation of the ribosome pathway has been observed in other pathogens during intracellular infection of macrophages, including Bordetella pertussis and Leishmania (Dillon et al., 2015; Petrácková et al., 2020). As well as a component of the stringent response, ribosomes are targets of antibiotics, so it is possible that downregulation of ribosome biogenesis may be a mechanism of bacterial defence against antibiotics. One such antibiotic which targets the bacterial ribosome is the macrolide azithromycin. A study by Taylor et al. demonstrated that long term azithromycin treatment reduced $H$. influenzae load and exacerbation risk in severe asthmatics (Taylor et al., 2019). However, this reduction was associated with increased carriage of antibiotic resistance genes by certain bacteria. Furthermore, Olszewska-Sosińska et al., found that persistent NTHi isolates recovered from macrophages obtained from azithromycin-treated children were not azithromycin resistant (Olszewska-Sosińska et al., 2016). As such, rather than active resistance to antibiotics conferred by specific antibiotic resistance genes, the maintenance of persister cells and drug tolerance may occur by other mechanisms, such as downregulation of ribosomes during intracellular persistence. These findings highlight the crucial importance of rapidly identifying and developing novel antimicrobials, given the global antimicrobial resistance crisis. The data set generated from this work provides a rich resource for exploration or screening of bacterial genes that may be associated with 
intracellular survival which may identify novel targets for antimicrobial therapeutics.

This work identified significant enrichment of various bacterial metabolic pathways, in agreement with a previous pioneering dual RNASeq study investigating NTHi-epithelial cell interactions (Baddal et al., 2015). Metabolic adaptations to changes in host substrate availability have been suggested to contribute to NTHi pathogenesis (Othman et al., 2014). In this current work, the bioC gene displayed the highest level of upregulation in the NTHi data set and was significantly upregulated compared to planktonic NTHi. Encoding for an $\mathrm{O}$-methyltransferase, bioC is involved in generating the pimeloyl acyl carrier protein (ACP) by the fatty acid synthesis pathway and can be used as a precursor for biotin synthesis (also known as vitamin H or B7) (Lin and Cronan, 2012). Biotin is a limited intracellular resource, thus intracellular pathogens able to scavenge or generate biotin in biotin-restricted environments could be better adapted to survive in an intracellular niche. This concept is supported by studies demonstrating the importance of biotin for survival and fitness of other intracellular pathogens (Yu et al., 2011; Napier et al., 2012; Sprenger et al., 2020). As biotin synthesis pathways are absent in humans, components of the biotin synthesis pathway could be attractive targets for therapeutics against intracellular pathogens. Determining whether NTHi intracellular persistence is dependent on the ability of NTHi to scavenge host biotin could identify biotin synthesis pathways as a potential therapeutic target.

In the current dataset, several genes previously reported to be important for NTHi oxidative stress responses (Harrison et al., 2007) were downregulated or not differentially expressed. This could suggest NTHi escape from macrophage intracellular killing mechanisms by $24 \mathrm{~h}$. In particular, the $d p s$ gene has been suggested to play a role in protecting NTHi from oxidative stress and has also been identified to be crucial for NTHi biofilm growth (Pang et al., 2012; Swords, 2012). The dps gene exhibited the highest decrease in expression at $24 \mathrm{~h}$, suggesting NTHi adaptation to intracellular residence within MDM is distinct to that of biofilm growth. Differences between NTHi lifestyles could have important implications for development of effective therapeutics. Although this work attempted to validate the results of the dual RNASeq transcriptomic analysis using planktonic NTHi by qPCR, one of the main limitations of this analysis is the absence of sequenced planktonic comparisons. Sequencing planktonic NTHi would have allowed for assessment of the differences in the transcriptomic profiles between planktonic and intracellular NTHi.

Additionally, as well as the previously discussed antibacterial functions, azithromycin also exhibits immunomodulatory functions, including antioxidant properties (Bergamini et al., 2009; Mal et al., 2013). However, dampening of oxidative stress responses in the airway may in fact promote NTHi persistence. NTHi utilises multiple strategies to promote a multifaceted defence response against oxidative stress, including regulation of dps (Harrison et al., 2012). As dps was downregulated at $24 \mathrm{~h}$ compared to $6 \mathrm{~h}$ in all three strains tested in this current work, it is possible that multiple strains of NTHi can overcome host oxidative stress responses to facilitate intracellular persistence. Thus, azithromycin-mediated dampening of antioxidant responses may contribute to NTHi persistence in the airway. Instead, the other immunomodulatory functions of azithromycin may be more important in reducing NTHi load, including increasing macrophage phagocytosis, upregulating expression of cell surface receptors (e.g. mannose receptor) and reducing pro-inflammatory cytokine levels (Hodge et al., 2006; Hodge et al., 2008; Euba et al., 2015). The AMAZES trial showed that despite a significant decrease in $H$. influenzae copy number, carriage of NTHi was still evident in some patients (Taylor et al., 2019). Thus, strain-dependent differences in NTHi adaptation to the lung environment may be responsible for the chronic airway colonisation by some strains; determining the transcriptomic profile of multiple diverse NTHi strains persisting despite azithromycin treatment would be informative.

NTHi strains are heterogeneous, with strain-dependent differences in macrophage persistence shown in this current work and that of Craig et al. (Craig et al., 2001). The seven strains of NTHi used for phylogenetic analysis were all clinical isolates cultured from either protected bronchial brushings, induced sputum or nasal brushings. However, strains did not cluster according to sample type. Although this is a small sample size, dissimilarity of NTHi strains derived from the same clinical source has also been shown in two larger studies (Erwin et al., 2008; De Chiara et al., 2014). Furthermore, two invasive NTHi strains (C188 and R2866) displayed increased ability to metabolise diverse substrates compared to a COPD isolate (Hi2019), whereas Hi2019 was better able to invade and reside within airway cells (Muda et al., 2019). This comparative analysis suggests differences in metabolic adaptations could underpin the ability of NTHi to persist in certain anatomical niches, further supported by the different levels of gene expression exhibited by the three diverse strains used in this current study. Thus, stratifying NTHi strains by transcriptomic adaptations during persistence could be a better method by which to identify strains better able to persist in vivo. However, a limitation of our model is that it is likely too acute to consider the long-term implications of NTHi colonisation and associated transcriptomic changes in the airway. Future investigations assessing the key gene targets identified in this analysis using macrophages isolated from individuals chronically colonised with NTHi will confirm the significance of transcriptomic adaptations to NTHi intracellular persistence within macrophages in the airway.

We recognise another limitation of our current work was the use of MDM to model lung macrophages. Although MDM have been extensively used and display similar phenotypes and responses to alveolar macrophages (Akagawa et al., 2006; Tudhope et al., 2008; Taylor et al., 2010), they are likely to not be completely reflective of NTHi-macrophage interactions in the airway, particularly given the reported functional impairments of macrophages from individuals with chronic respiratory disease (Taylor et al., 2010; Staples et al., 2012; Berenson et al., 2013; Liang et al., 2014; Belchamber et al., 2019). Moreover, lung macrophages in COPD consist of various subpopulations, which also differ from control lung macrophages in terms of pro-inflammatory and 
phagocytic ability (Dewhurst et al., 2017). As such, it is possible that a specific sub-population of macrophages may function as a preferred niche for intracellular persistence. Notably, dual RNASeq analysis comparing responses of alveolar and interstitial macrophages (AM and IM, respectively) to infection with Mtb found that Mtb growth was more restricted in IM, which demonstrated a more pro-inflammatory response than AM (Pisu et al., 2020). Thus, it would be important to confirm the transcriptomic findings in this study using lung macrophages from chronic respiratory disease patients colonised with NTHi.

\section{FINAL CONCLUSIONS}

In summary, this work demonstrates that despite significant enrichment of immune processes and intracellular pathways by MDM, NTHi was able to persist for the duration of our experiments. Thus, macrophages may act as a protected niche within the airway, promoting NTHi colonisation, especially in chronic respiratory diseases that already have evidence of macrophage dysfunction. Furthermore, this persistence may contribute to the dysregulated immune response in chronic respiratory disease, as upregulation of macrophage proinflammatory responses, including mediators of other inflammatory immune cells, were detected in response to NTHi persistence. Analysis of NTHi gene expression during persistence revealed metabolic adaptations which may be crucial for bacterial persistence. However, further functional work is needed to confirm the importance of genes and pathways before they are deemed key targets to be taken forward into drug development pipelines for validation. Nonetheless, this work provides a rich transcriptomic resource for exploration of hostpathogen interactions, which could unveil novel gene targets for therapeutic interventions to reduce the burden of NTHi in the airway.

\section{DATA AVAILABILITY STATEMENT}

The original contributions presented in the study are publicly available. This data can be found here: GSE180166 (http://ncbi. nlm.nih.gov.geo/).

\section{REFERENCES}

Ahearn, C. P., Gallo, M. C., and Murphy, T. F. (2017). Insights on Persistent Airway Infection by Non-Typeable Haemophilus Influenzae in Chronic Obstructive Pulmonary Disease. Pathog. Dis. 75 (4), 1-18. doi: 10.1093/femspd/ftx042

Ahrén, I. L., Williams, D. L., Rice, P. J., Forsgren, A., and Riesbeck, K. (2001). The Importance of a $\beta$-Glucan Receptor in the Nonopsonic Entry of Nontypeable Haemophilus Influenzae Into Human Monocytic and Epithelial Cells. J. Infect. Dis. 184 (2), 150-158. doi: 10.1086/322016

Akagawa, K. S., Komuro, I., Kanazawa, H., Yamazaki, T., Mochida, K., and Kishi, F. (2006). Functional Heterogeneity of Colony-Stimulating Factor-Induced Human Monocyte-Derived Macrophages. Respirology 11 (Suppl. 1), 27-34. doi: 10.1111/j.1440-1843.2006.00805.x

\section{ETHICS STATEMENT}

The studies involving human participants were reviewed and approved by Hampshire A Research Ethics Committee (13/SC/ 0416). The patients/participants provided their written informed consent to participate in this study.

\section{AUTHOR CONTRIBUTIONS}

JA and KS conceptualized the project. JA, AH, DC, MC and KS contributed to methodology. JA and $\mathrm{AH}$ undertook the formal analysis. KS administered the project. JA performed the investigation. DC, MC, TW and KS provided resources and acquired funding. DC, MC, TW and KS supervised the project. JA, AH and DC curated the data. JA and KS wrote the original draft. All authors contributed to the article and approved the submitted version.

\section{FUNDING}

This work was primarily funded by an Asthma UK studentship award (AUK-PHD-2016-363). We also gratefully acknowledge the support of the Southampton AAIR charity in funding this work. The funders had no role in study design, data collection and interpretation, or the decision to submit the work for publication.

\section{ACKNOWLEDGMENTS}

The authors would like to express gratitude to all the volunteers who kindly provided blood samples for this study. The authors also wish to thank Richard Jewell and Dr Carolann McGuire of the University of Southampton, Faculty of Medicine Flow Cytometry Unit and Dr Alastair Watson for his help in preparing the manuscript for submission.

\section{SUPPLEMENTARY MATERIAL}

The Supplementary Material for this article can be found online at: https://www.frontiersin.org/articles/10.3389/fcimb.2021.723481/ full\#supplementary-material

Anders, S., and Huber, W. (2010). Differential Expression Analysis for Sequence Count Data. Genome Biol. 11 (10), R106. doi: 10.1186/gb2010-11-10-r106

Anders, S., Pyl, P. T., and Huber, W. (2015). HTSeq-A Python Framework to Work With High-Throughput Sequencing Data. Bioinformatics 31 (2), 166 169. doi: 10.1093/bioinformatics/btu638

Arango Duque, G., and Descoteaux, A. (2014). Macrophage Cytokines: Involvement in Immunity and Infectious Diseases. Front. Immunol. 5, 491. doi: 10.3389/fimmu.2014.00491

Baddal, B., Muzzi, A., Censini, S., Calogero, R. A., Torricelli, G., Guidotti, S., et al. (2015). Dual RNA-Seq of Nontypeable Haemophilus Influenzae and Host Cell Transcriptomes Reveals Novel Insights Into Host-Pathogen Cross Talk. mBio 6 (6), 1-13. doi: 10.1128/mBio.01765-15 
Belchamber, K. B. R., Singh, R., Batista, C. M., Whyte, M. K., Dockrell, D. H., Kilty, I., et al. (2019). Defective Bacterial Phagocytosis Is Associated With Dysfunctional Mitochondria in COPD Macrophages. Eur. Respir. J. 54 (4), 1802244. doi: 10.1183/ 13993003.02244-2018

Berenson, C. S., Garlipp, M. A., Grove, L. J., Maloney, J., and Sethi, S. (2006). Impaired Phagocytosis of Nontypeable Haemophilus Influenzae by Human Alveolar Macrophages in Chronic Obstructive Pulmonary Disease. J. Infect. Dis. 194 (10), 1375-1384. doi: 10.1086/508428

Berenson, C. S., Kruzel, R. L., Eberhardt, E., and Sethi, S. (2013). Phagocytic Dysfunction of Human Alveolar Macrophages and Severity of Chronic Obstructive Pulmonary Disease. J. Infect. Dis. 208 (12), 2036-2045. doi: 10.1093/ infdis/jit400

Bergamini, G., Cigana, C., Sorio, C., Della Peruta, M., Pompella, A., Corti, A., et al. (2009). Effects of Azithromycin on Glutathione S-Transferases in Cystic Fibrosis Airway Cells. Am. J. Respir. Cell Mol. Biol. 41 (2), 199-206. doi: 10.1165/ rcmb.2008-00130C

Byrne, A. J., Mathie, S. A., Gregory, L. G., and Lloyd, C. M. (2015). Pulmonary Macrophages: Key Players in the Innate Defence of the Airways. Thorax 70 (12), 1189-1196. doi: 10.1136/thoraxjnl-2015-207020

Chen, J., Bardes, E. E., Aronow, B. J., and Jegga, A. G. (2009). ToppGene Suite for Gene List Enrichment Analysis and Candidate Gene Prioritization. Nucleic Acids Res. 37 (SUPPL. 2), 305-311. doi: 10.1093/nar/gkp427

Clementi, C. F., and Murphy, T. F. (2011). Non-Typeable Haemophilus Influenzae Invasion and Persistence in the Human Respiratory Tract. Front. Cell. Infect. Microbiol. 1, 1. doi: 10.3389/fcimb.2011.00001

Cooper, G. E., Ostridge, K., Khakoo, S. I., Wilkinson, T. M. A., and Staples, K. J. (2018). Human CD49a + Lung Natural Killer Cell Cytotoxicity in Response to Influenza A Virus. Front. Immunol. 9, 1671. doi: 10.3389/fimmu.2018.01671

Coughtrie, A. L., Morris, D. E., Anderson, R., Begum, N., Cleary, D. W., Faust, S. N., et al. (2018). Ecology and Diversity in Upper Respiratory Tract Microbial Population Structures From a Cross-Sectional Community Swabbing Study. J. Med. Microbiol. 67 (8), 1096-1108. doi: 10.1099/jmm.0.000773

Craig, J. E., Cliffe, A., Garnett, K., and High, N. J. (2001). Survival of Nontypeable Haemophilus Influenzae in Macrophages. FEMS Microbiol. Lett. 203 (1), 5561. doi: 10.1016/S0378-1097(01)00328-7

Craig, J. E., Nobbs, A., and High, N. J. (2002). The Extracytoplasmic Sigma Factor, ৎe, Is Required for Intracellular Survival of Nontypeable Haemophilus Influenzae in J774 Macrophages. Infect. Immun. 70 (2), 708-715. doi: 10.1128/IAI.70.2.708-715.2002

De Chiara, M., Hood, D., Muzzi, A., Pickard, D. J., Perkins, T., Pizza, M., et al. (2014). Genome Sequencing of Disease and Carriage Isolates of Nontypeable Haemophilus Influenzae Identifies Discrete Population Structure. Proc. Natl. Acad. Sci. U. S. A 111 (14), 5439-5444. doi: 10.1073/pnas.1403353111

Dewhurst, J. A., Lea, S., Hardaker, E., Dungwa, J. V., Ravi, A. K., and Singh, D. (2017). Characterisation of Lung Macrophage Subpopulations in COPD Patients and Controls. Sci. Rep. 7 (1), 7143. doi: 10.1038/s41598-01707101-2

Dillon, L. A. L., Suresh, R., Okrah, K., Corrada Bravo, H., Mosser, D. M., and ElSayed, N. M. (2015). Simultaneous Transcriptional Profiling of Leishmania Major and Its Murine Macrophage Host Cell Reveals Insights Into HostPathogen Interactions. BMC Genomics 16 (1), 1108-1108. doi: 10.1186/ s12864-015-2237-2

Dobin, A., Davis, C. A., Schlesinger, F., Drenkow, J., Zaleski, C., Jha, S., et al. (2013). STAR: Ultrafast Universal RNA-Seq Aligner. Bioinformatics 29 (1), 1521. doi: 10.1093/bioinformatics/bts635

Erwin, A. L., Sandstedt, S. A., Bonthuis, P. J., Geelhood, J. L., Nelson, K. L., Unrath, W. C. T., et al. (2008). Analysis of Genetic Relatedness of Haemophilus Influenzae Isolates by Multilocus Sequence Typing. J. Bacteriol. 190 (4), 1473-1483. doi: 10.1128/JB.01207-07

Erwin, A. L., and Smith, A. L. (2007). Nontypeable Haemophilus Influenzae: Understanding Virulence and Commensal Behavior. Trends Microbiol. 15 (8), 355-362. doi: 10.1016/j.tim.2007.06.004

Euba, B., Moleres, J., Viadas, C., Barberán, M., Caballero, L., Grilló, M. J., et al. (2015). Relationship Between Azithromycin Susceptibility and Administration Efficacy for Nontypeable Haemophilus Influenzae Respiratory Infection. Antimicrob. Agents Chemother. 59 (5), 2700-2712. doi: 10.1128/AAC.04447-14

Farley, M. M., Stephens, D. S., Mulks, M. H., Cooper, M. D., Bricker, J. V., Mirra, S. S., et al. (1986). Pathogenesis of IgAl Protease-Producing and-Nonproducing
Haemophilus Influenzae in Human Nasopharyngeal Organ Cultures. J. Inf. Dis. 154 (5), 752-759. doi: 10.1093/infdis/154.5.752

Finney, L. J., Ritchie, A., Pollard, E., Johnston, S. L., and Mallia, P. (2014). Lower Airway Colonization and Inflammatory Response in COPD: A Focus on Haemophilus Influenza. Int. J. COPD 9, 1119-1132. doi: 10.2147/ COPD.S54477

Forsgren, J., Samuelson, A., Ahlin, A., Jonasson, J., Rynnel-Dagoo, B., and Lindberg, A. (1994). Haemophilus Influenzae Resides and Multiplies Intracellularly in Human Adenoid Tissue as Demonstrated by In Situ Hybridization and Bacterial Viability Assay. Infect. Immun. 62 (2), 673-679. doi: 10.1128/iai.62.2.673-679.1994

Gallo, M. C., Kirkham, C., Eng, S., Bebawee, R. S., Kong, Y., Pettigrew, M. M., et al. (2018). Changes in IgA Protease Expression Are Conferred by Changes in Genomes During Persistent Infection by Nontypeable Haemophilus Influenzae in Chronic Obstructive Pulmonary Disease. Infect. Immun. 86 (8), e00313e00318. doi: 10.1128/IAI.00313-18

Ge, S. X., Jung, D., Jung, D., and Yao, R. (2020). ShinyGO: A Graphical Gene-Set Enrichment Tool for Animals and Plants. Bioinformatics 36 (8), 2628-2629. doi: 10.1093/bioinformatics/btz931

Green, B. J., Wiriyachaiporn, S., Grainge, C., Rogers, G. B., Kehagia, V., Lau, R., et al. (2014). Potentially Pathogenic Airway Bacteria and Neutrophilic Inflammation in Treatment Resistant Severe Asthma. PloS One 9 (6), e100645. doi: 10.1371/journal.pone. 0100645

Harrison, A., Bakaletz, L. O., and Munson, R. S. (2012). Haemophilus Influenzae and Oxidative Stress. Front. Cell. Infectt. Microbiol. 2, 40. doi: 10.3389/ fcimb.2012.00040. Frontiers Media SA.

Harrison, A., Ray, W. C., Baker, B. D., Armbruster, D. W., Bakaletz, L. O., and Munson, R. S. (2007). The OxyR Regulon in Nontypeable Haemophilus Influenzae. J. Bacteriol. 189 (3), 1004-1012. doi: 10.1128/JB.01040-06

Hersch, S. J., and Navarre, W. W. (2020). The Salmonella LysR Family Regulator RipR Activates the SPI-13-Encoded Itaconate Degradation Cluster. Infect. Immun. 88 (10), e00303-20. doi: 10.1128/IAI.00303-20

Hodge, S., Hodge, G., Brozyna, S., Jersmann, H., Holmes, M., and Reynolds, P. N. (2006). Azithromycin Increases Phagocytosis of Apoptotic Bronchial Epithelial Cells by Alveolar Macrophages. Eur. Respir. J. 28 (3), 486-495. doi: 10.1183/ 09031936.06.00001506

Hodge, S., Hodge, G., Jersmann, H., Matthews, G., Ahern, J., Holmes, M., et al. (2008). Azithromycin Improves Macrophage Phagocytic Function and Expression of Mannose Receptor in Chronic Obstructive Pulmonary Disease. Am. J. Respir. Crit. Care Med. 178 (2), 139-148. doi: 10.1164/rccm.200711$1666 \mathrm{OC}$

Hoffmann, E., Machelart, A., Belhaouane, I., Deboosere, N., Pauwels, A. M., SaintAndré, J. P., et al. (2019). Irgl Controls Immunometabolic Host Response and Restricts Intracellular Mycobacterium Tuberculosis Infection: Immunometabolic Host Response to Tuberculosis. BioRxiv 761551. doi: 10.1101/761551

Iikura, M., Hojo, M., Koketsu, R., Watanabe, S., Sato, A., Chino, H., et al. (2015). The Importance of Bacterial and Viral Infections Associated With Adult Asthma Exacerbations in Clinical Practice. PloS One 10 (4), e0123584. doi: 10.1371 /journal.pone. 0123584

Kaufmann, S. H. E., and Dorhoi, A. (2016). Molecular Determinants in PhagocyteBacteria Interactions. Immunity 44 (3), 476-491. doi: 10.1016/j.immuni. 2016.02.014

Kim, B.-H., Shenoy, A. R., Kumar, P., Das, R., Tiwari, S., and MacMicking, J. D. (2011). A Family of IFN- $\gamma$-Inducible 65-kD GTPases Protects Against Bacterial Infection. Sci. (New York N. Y.) 332 (6030), 717-721. doi: 10.1126/ science. 1201711

King, P. (2012). Haemophilus Influenzae and the Lung (Haemophilus and the Lung). Clin. Trans. Med. 1 (1), 10. doi: 10.1186/2001-1326-1-10

King, P., Ngui, J., Oppedisano, F., Robins-Browne, R., Holmes, P., and Holdsworth, S. (2008). Effect of Interferon Gamma and CD40 Ligation on Intracellular Monocyte Survival of Nontypeable Haemophilus Influenzae. APMIS: Acta Pathol. Microbiol. Immunol. Scand. 116 (12), 1043-1049. doi: 10.1111/j.1600-0463.2008.01078.x

Kirkham, L.-A. S., Corscadden, K. J., Wiertsema, S. P., Currie, A. J., and Richmond, P. C. (2013). A Practical Method for Preparation of Pneumococcal and Nontypeable Haemophilus Influenzae Inocula That Preserves Viability and Immunostimulatory Activity. BMC Res. Notes 6 (1), 522. doi: $10.1186 / 1756-0500-6-522$ 
Kucera, M., Isserlin, R., Arkhangorodsky, A., and Bader, G. D. (2016). AutoAnnotate: A Cytoscape App for Summarizing Networks With Semantic Annotations. F1000Research 5, 1717. doi: 10.12688/f1000research.9090.1

Langmead, B., and Salzberg, S. L. (2012). Fast Gapped-Read Alignment With Bowtie 2. Nat. Methods 9 (4), 357-359. doi: 10.1038/nmeth.1923

Lee, C. G. L., Jenkins, N. A., Gilbert, D. J., Copeland, N. G., and O’Brien, W. E. (1995). Cloning and Analysis of Gene Regulation of a Novel LPS-Inducible cDNA. Immunogenetics 41 (5), 263-270. doi: 10.1007/BF00172150

Liang, Z., Zhang, Q., Thomas, C. M. R., Chana, K. K., Gibeon, D., Barnes, P. J., et al. (2014). Impaired Macrophage Phagocytosis of Bacteria in Severe Asthma. Respir. Res. 15 (1), 72. doi: 10.1186/1465-9921-15-72

Lin, S., and Cronan, J. E. (2012). The BioC O-Methyltransferase Catalyzes Methyl Esterification of Malonyl-Acyl Carrier Protein, an Essential Step in Biotin Synthesis. J. Biol. Chem. 287 (44), 37010-37020. doi: 10.1074/jbc.M112.410290

Mal, P., Dutta, K., Bandyopadhyay, D., Basu, A., Khan, R., and Bishayi, B. (2013). Azithromycin in Combination With Riboflavin Decreases the Severity of Staphylococcus Aureus Infection Induced Septic Arthritis by Modulating the Production of Free Radicals and Endogenous Cytokines. Inflamm. Res. 62 (3), 259-273. doi: 10.1007/s00011-012-0574-z

Marsh, J. W., Humphrys, M. S., and Myers, G. S. A. (2017). A Laboratory Methodology for Dual RNA-Sequencing of Bacteria and Their Host Cells In Vitro. Front. Microbiol. 8:1830 (SEP). doi: 10.3389/fmicb.2017.01830

Martí-Lliteras, P., Regueiro, V., Morey, P., Hood, D. W., Saus, C., Sauleda, J., et al. (2009). Nontypeable Haemophilus Influenzae Clearance by Alveolar Macrophages Is Impaired by Exposure to Cigarette Smoke. Infect. Immun. 77 (10), 4232-4242. doi: 10.1128/IAI.00305-09

Mayhew, D., Devos, N., Lambert, C., Brown, J. R., Clarke, S. C., Kim, V. L., et al. (2018). Longitudinal Profiling of the Lung Microbiome in the AERIS Study Demonstrates Repeatability of Bacterial and Eosinophilic COPD Exacerbations. Thorax 73 (5), 422-430. doi: 10.1136/thoraxjnl-2017-210408

McCann, J. R., Mason, S. N., Auten, R. L., St. Geme, J. W., and Seed, P. C. (2016). Early-Life Intranasal Colonization With Nontypeable Haemophilus Influenzae Exacerbates Juvenile Airway Disease in Mice. Infect. Immun. 84 (7), 20222030. doi: 10.1128/IAI.01539-15

McKendry, R. T., Spalluto, C. M., Burke, H., Nicholas, B., Cellura, D., AlShamkhani, A., et al. (2016). Dysregulation of Antiviral Function of CD8+ T Cells in the Chronic Obstructive Pulmonary Disease Lung. Role of the PD-1PD-L1 Axis. Am. J. Respir. Crit. Care Med. 193 (6), 642-651. doi: 10.1164/ rccm.201504-0782OC

Merico, D., Isserlin, R., Stueker, O., Emili, A., and Bader, G. D. (2010). Enrichment Map: A Network-Based Method for Gene-Set Enrichment Visualization and Interpretation. PloS One 5 (11), e13984. doi: 10.1371/journal.pone.0013984

Meunier, E., Dick, M. S., Dreier, R. F., Schürmann, N., Broz, D. K., Warming, S., et al. (2014). Caspase-11 Activation Requires Lysis of Pathogen-Containing Vacuoles by IFN-Induced GTPases. Nature 509 (7500), 366-370. doi: 10.1038/ nature13157

Meunier, E., Wallet, P., Dreier, R. F., Costanzo, S., Anton, L., Rühl, S., et al. (2015). Guanylate-Binding Proteins Promote Activation of the AIM2 Inflammasome During Infection With Francisella Novicida. Nat. Immunol. 16 (5), 476-484. doi: $10.1038 /$ ni.3119

Michelucci, A., Cordes, T., Ghelfi, J., Pailot, A., Reiling, N., Goldmann, O., et al. (2013). Immune-Responsive Gene 1 Protein Links Metabolism to Immunity by Catalyzing Itaconic Acid Production. Proc. Natl. Acad. Sci. U. S. A 110 (19), 7820-7825. doi: 10.1073/pnas.1218599110

Morey, P., Cano, V., Martí-Lliteras, P., López-Gómez, A., Regueiro, V., Saus, C., et al. (2011). Evidence for a Non-Replicative Intracellular Stage of Nontypable Haemophilus Influenzae in Epithelial Cells. Microbiol. (Reading Engl) 157 (Pt 1), 234-250. doi: 10.1099/mic.0.040451-0

Mosser, D. M., and Edwards, J. P. (2008). Exploring the Full Spectrum of Macrophage Activation. Nat. Rev. Immunol. 8 (12), 958-969. doi: 10.1038/ nri2448

Muda, N. M., Nasreen, M., Dhouib, R., Hosmer, J., Hill, J., Mahawar, M., et al. (2019). Metabolic Analyses Reveal Common Adaptations in Two Invasive Haemophilus Influenzae Strains. Pathog. Dis. 77 (2), 15. doi: 10.1093/femspd/ $\mathrm{ftz} 015$

Mulay, A., Akram, K. M., Williams, D., Armes, H., Russell, C., Hood, D., et al. (2016). An In Vitro Model of Murine Middle Ear Epithelium. DMM. Dis. Models Mech. 9 (11), 1405-1417. doi: 10.1242/dmm.026658
Murphy, T. F., Brauer, A. L., Schiffmacher, A. T., and Sethi, S. (2004). Persistent Colonization by Haemophilus Influenzae in Chronic Obstructive Pulmonary Disease. Am. J. Respir. Crit. Care Med. 170 (3), 266-272. doi: 10.1164/ rccm.200403-354oc

Nair, S., Huynh, J. P., Lampropoulou, V., Loginicheva, E., Esaulova, E., Gounder, A. P., et al. (2018). Irg1 Expression in Myeloid Cells Prevents Immunopathology During M. Tuberculosis Infection. J. Exp. Med. 215 (4), 1035-1045. doi: 10.1084/jem.20180118

Napier, B. A., Meyer, L., Bina, J. E., Miller, M. A., Sjöstedt, A., and Weiss, D. S. (2012). Link Between Intraphagosomal Biotin and Rapid Phagosomal Escape in Francisella. Proc. Natl. Acad. Sci. U. S. A 109 (44), 18084-18089. doi: $10.1073 /$ pnas. 1206411109

Ogger, P. P., Albers, G. J., Hewitt, R. J., O'Sullivan, B. J., Powell, J. E., Calamita, E., et al. (2020). Itaconate Controls the Severity of Pulmonary Fibrosis. Sci. Immunol. 5 (52), 282-295. doi: 10.1126/sciimmunol.abc1884

Olszewska-Sosińska, O., Zielnik-Jurkiewicz, B., Stępińska, M., Antos-Bielska, M., Lau-Dworak, M., Kozłowska, K., et al. (2016). Persistence of Non-Typeable Haemophilus Influenzae in the Pharynx of Children With Adenotonsillar Hypertrophy After Treatment With Azithromycin. Pathog. Dis. 74 (1), ftv106. doi: 10.1093/femspd/ftv106

O'Neill, L. A. J., and Artyomov, M. N. (2019). Itaconate: The Poster Child of Metabolic Reprogramming in Macrophage Function. Nat. Rev. Immunol. 19, 273-281. doi: 10.1038/s41577-019-0128-5. Nature Publishing Group.

Osman, K. L., Jefferies, J. M. C., Woelk, C. H., Devos, N., Pascal, T. G., Mortier, M.C., et al. (2018). Patients With Chronic Obstructive Pulmonary Disease Harbour a Variation of Haemophilus Species. Sci. Rep. 8 (1), 14734. doi: 10.1038/s41598-018-32973-3

Othman, D. S. M. P., Schirra, H., McEwan, A. G., and Kappler, U. (2014). Metabolic Versatility in Haemophilus Influenzae: A Metabolomic and Genomic Analysis. Front. Microbiol. 5, 69. doi: 10.3389/fmicb.2014.00069

Pang, B., Hong, W., Kock, N. D., and Swords, W. E. (2012). Dps Promotes Survival of Nontypeable Haemophilus Influenzae in Biofilm Communities In Vitro and Resistance to Clearance In Vivo. Front. Cell. Infect. Microbiol. 2, 58. doi: $10.3389 /$ fcimb.2012.00058

Petráčková, D., Farman, M. R., Amman, F., Linhartová, I., Dienstbier, A., Kumar, D., et al. (2020). Transcriptional Profiling of Human Macrophages During Infection With Bordetella Pertussis. RNA Biol. 17 (5), 731-742. doi: 10.1080/ 15476286.2020.1727694

Pettigrew, M. M., Ahearn, C. P., Gent, J. F., Kong, Y., Gallo, M. C., Munro, J. B., et al. (2018). Haemophilus Influenzae Genome Evolution During Persistence in the Human Airways in Chronic Obstructive Pulmonary Disease. Proc. Natl. Acad. Sci. U. S. A. 115 (14), E3256-E3265. doi: 10.1073/pnas.1719654115

Pisu, D., Huang, L., Grenier, J. K., and Russell, D. G. (2020). Dual RNA-Seq of Mtb-Infected Macrophages In Vivo Reveals Ontologically Distinct HostPathogen Interactions. Cell Rep. 30 (2), 335-350.e4. doi: 10.1016/ j.celrep.2019.12.033

Riquelme, S. A., Liimatta, K., Wong Fok Lung, T., Fields, B., Ahn, D., Chen, D., et al. (2020). Pseudomonas Aeruginosa Utilizes Host-Derived Itaconate to Redirect Its Metabolism to Promote Biofilm Formation. Cell Metab. 31 (6), 1091-1106.e6. doi: 10.1016/j.cmet.2020.04.017

Robinson, M. D., McCarthy, D. J., and Smyth, G. K. (2009). Edger: A Bioconductor Package for Differential Expression Analysis of Digital Gene Expression Data. Bioinformatics 26 (1), 139-140. doi: 10.1093/bioinformatics/btp616

Román, F., Cantón, R., Pérez-Vázquez, M., Baquero, F., and Campos, J. (2004). Dynamics of Long-Term Colonization of Respiratory Tract by Haemophilus Influenzae in Cystic Fibrosis Patients Shows a Marked Increase in Hypermutable Strains. J. Clin. Microbiol. 42 (4), 1450-1459. doi: 10.1128/JCM.42.4.1450-1459.2004

Santos, J. C., Boucher, D., Schneider, L. K., Demarco, B., Dilucca, M., Shkarina, K., et al. (2020). Human GBP1 Binds LPS to Initiate Assembly of a Caspase-4 Activating Platform on Cytosolic Bacteria. Nat. Commun. 11 (1), 3276. doi: $10.1038 / \mathrm{s} 41467-020-16889-\mathrm{z}$

Sasikaran, J., Ziemski, M., Zadora, P. K., Fleig, A., and Berg, I. A. (2014). Bacterial Itaconate Degradation Promotes Pathogenicity. Nat. Chem. Biol. 10 (5), 371377. doi: 10.1038/nchembio. 1482

Singhania, A., Wallington, J. C., Smith, C. G., Horowitz, D., Staples, K. J., Howarth, P. H., et al. (2018). Multitissue Transcriptomics Delineates the Diversity of Airway T Cell Functions in Asthma. Am. J. Respir. Cell Mol. Biol. 58, 261-270). doi: 10.1165/rcmb.2017-0162OC. American Thoracic Society. 
Singh, R., Belchamber, K. B. R., Fenwick, P. S., Chana, K., Donaldson, G., Wedzicha, J. A., et al. (2021). Defective Monocyte-Derived Macrophage Phagocytosis Is Associated With Exacerbation Frequency in COPD. Respir. Res. 22 (1), 113. doi: 10.1186/s12931-021-01718-8

Song, C., Luo, L., Lei, Z., Li, B., Liang, Z., Liu, G., et al. (2008). IL-17-Producing Alveolar Macrophages Mediate Allergic Lung Inflammation Related to Asthma. J. Immunol. 181 (9), 6117-6124. doi: 10.4049/jimmunol.181.9.6117

Sprenger, M., Hartung, T. S., Allert, S., Wisgott, S., Niemiec, M. J., Graf, K., et al. (2020). Fungal Biotin Homeostasis is Essential for Immune Evasion After Macrophage Phagocytosis and Virulence. Cell. Microbiol. 22 (7), e13197. doi: $10.1111 / \mathrm{cmi} .13197$

Staples, K. J., Hinks, T. S. C., Ward, J. A., Gunn, V., Smith, C., and Djukanović, R. (2012). Phenotypic Characterization of Lung Macrophages in Asthmatic Patients: Overexpression of CCL17. J. Allergy Clin. Immunol. 130 (6), 14041412.e7. doi: 10.1016/j.jaci.2012.07.023

Staples, K. J., Nicholas, B., McKendry, R. T., Spalluto, C. M., Wallington, J. C., Bragg, C. W., et al. (2015). Viral Infection of Human Lung Macrophages Increases PDL1 Expression via Ifnß. PloS One 10 (3), e0121527. doi: 10.1371/ journal.pone.0121527

Swords, W. E. (2012). Nontypeable Haemophilus Influenzae Biofilms: Role in Chronic Airway Infections. Front. Cell. Infect. Microbiol. 2:97. doi: 10.3389/ fcimb.2012.00097

Taylor, A. E., Finney-Hayward, T. K., Quint, J. K., Thomas, C. M. R., Tudhope, S. J., Wedzicha, J. A., et al. (2010). Defective Macrophage Phagocytosis of Bacteria in COPD. Eur. Respir. J. 35 (5), 1039-1047. doi: 10.1183/09031936.00036709

Taylor, S. L., Leong, L. E. X., Mobegi, F. M., Choo, J. M., Wesselingh, S., Yang, I. A., et al. (2019). Long-Term Azithromycin Reduces Haemophilus Influenzae and Increases Antibiotic Resistance in Severe Asthma. Am. J. Respir. Crit. Care Med. 200 (3), 309-317. doi: 10.1164/rccm.201809-1739OC

Treangen, T. J., Ondov, B. D., Koren, S., and Phillippy, A. M. (2014). The Harvest Suite for Rapid Core-Genome Alignment and Visualization of Thousands of Intraspecific Microbial Genomes. Genome Biol. 15 (11), 524. doi: 10.1186/ s13059-014-0524-x

Tudhope, S. J., Finney-Hayward, T. K., Nicholson, A. G., Mayer, R. J., Barnette, M. S., Barnes, P. J., et al. (2008). Different Mitogen-Activated Protein KinaseDependent Cytokine Responses in Cells of the Monocyte Lineage. J. Pharmacol. Exp. Ther. 324 (1), 306-312. doi: 10.1124/jpet.107.127670

Van Eldere, J., Slack, M. P. E., Ladhani, S., and Cripps, A. W. (2014). NonTypeable Haemophilus Influenzae, an Under-Recognised Pathogen. Lancet Infect. Dis. 14 (12), 1281-1292. doi: 10.1016/S1473-3099(14)70734-0

Wallington, J. C., Williams, A. P., Staples, K. J., and Wilkinson, T. M. A. (2018). IL-12 and IL-7 Synergize to Control Mucosal-Associated Invariant T-Cell Cytotoxic Responses to Bacterial Infection. J. Allergy Clin. Immunol. 141 (6), 2182-2195.e6. doi: 10.1016/j.jaci.2017.08.009

Wilkinson, T. M. A., Aris, E., Bourne, S., Clarke, S. C., Peeters, M., Pascal, T. G., et al. (2017). A Prospective, Observational Cohort Study of the Seasonal Dynamics of Airway Pathogens in the Aetiology of Exacerbations in COPD. Thorax 72 (10), 919-927. doi: 10.1136/thoraxjnl-2016-209023
Wilson, D. N., and Nierhaus, K. H. (2007). The Weird and Wonderful World of Bacterial Ribosome Regulation. Crit. Rev. Biochem. Mol. Biol. 42 (3), 187-219. doi: $10.1080 / 10409230701360843$

Wood, L. G., Simpson, J. L., Hansbro, P. M., and Gibson, P. G. (2010). Potentially Pathogenic Bacteria Cultured From the Sputum of Stable Asthmatics Are Associated With Increased 8-Isoprostane and Airway Neutrophilia. Free Radical Res. 44 (2), 146-154. doi: 10.3109/10715760903362576

Yadav, M. C., Chakraborti, A., Ray, P., Sapru, S., Majumdar, S., and Narang, A. (2003). Rapid Detection of Haemophilus Influenzae by Hel Gene Polymerase Chain Reaction. Lett. Appl. Microbiol. 37 (3), 190-195. doi: 10.1046/j.1472765X.2003.01342.x

Yang, X., Wang, Y., Zhao, S., Wang, R., and Wang, C. (2018). Long-Term Exposure to Low-Dose Haemophilus Influenzae During Allergic Airway Disease Drives a Steroid-Resistant Neutrophilic Inflammation and Promotes Airway Remodeling. Oncotarget 9 (38), 24898-24913. doi: 10.18632/oncotarget.24653

Yu, J., Niu, C., Wang, D., Li, M., Teo, W., Sun, G., et al. (2011). MMAR_2770, a New Enzyme Involved in Biotin Biosynthesis, Is Essential for the Growth of Mycobacterium Marinum in Macrophages and Zebrafish. Microbes Infect. 13 (1), 33-41. doi: 10.1016/j.micinf.2010.08.010

Zhang, Q., Cox, M., Liang, Z., Brinkmann, F., Cardenas, P. A., Duff, R., et al. (2016). Airway Microbiota in Severe Asthma and Relationship to Asthma Severity and Phenotypes. PloS One 11 (4), 1-16. doi: 10.1371/journal.pone.0152724

Conflict of Interest: DC reports that he was a post-doctoral researcher on projects funded by Pfizer and GSK between April 2014 and October 2017. TW reports grants and personal fees from AstraZeneca, personal fees and other from MMH, grants and personal fees from GSK, personal fees from BI, and grants and personal fees from Synairgen, outside the submitted work. KS reports grants from AstraZeneca, outside the submitted work.

The remaining authors declare that the research was conducted in the absence of any commercial or financial relationships that could be construed as a potential conflict of interest.

Publisher's Note: All claims expressed in this article are solely those of the authors and do not necessarily represent those of their affiliated organizations, or those of the publisher, the editors and the reviewers. Any product that may be evaluated in this article, or claim that may be made by its manufacturer, is not guaranteed or endorsed by the publisher.

Copyright (C) 2021 Ackland, Heinson, Cleary, Christodoulides, Wilkinson and Staples. This is an open-access article distributed under the terms of the Creative Commons Attribution License (CC BY). The use, distribution or reproduction in other forums is permitted, provided the original author(s) and the copyright owner(s) are credited and that the original publication in this journal is cited, in accordance with accepted academic practice. No use, distribution or reproduction is permitted which does not comply with these terms. 